CLNS 93/1204

IP-ASTP-15-93

May, 1993

\title{
Heavy Quark and Chiral Symmetry Predictions for Semileptonic Decays $\bar{B} \rightarrow D\left(D^{*}\right) \pi \ell \bar{\nu}$
}

\author{
Hai-Yang Cheng ${ }^{a, d}$, Chi-Yee Cheung ${ }^{a}$, William Dimm ${ }^{c}$, Guey-Lin Lin ${ }^{a}$, \\ Y. C. Lin ${ }^{b}$, Tung-Mow Yan ${ }^{a, c}$, and Hoi-Lai Yu ${ }^{a}$ \\ ${ }^{a}$ Institute of Physics, Academia Sinica, Taipei, \\ Taiwan 11529, Republic of China \\ ${ }^{b}$ Physics Department, National Central University, Chung-li, \\ Taiwan 32054, Republic of China \\ ${ }^{c}$ Floyd R. Newman Laboratory of Nuclear Studies, Cornell University \\ Ithaca, New York 14853, USA \\ ${ }^{d}$ Institute for Theoretical Physics, State University of New York \\ Stony Brook, New York 11794, USA
}

\begin{abstract}
We study in detail the prediction for the semileptonic decays $\bar{B} \rightarrow D\left(D^{*}\right) \pi \ell \bar{\nu}$ by heavy quark and chiral symmetry. The branching ratio for $\bar{B} \rightarrow D \pi \ell \bar{\nu}$ is quite significant, as big as $(0.5-1) \%$. The branching ratio for $\bar{B} \rightarrow D^{*} \pi \ell \bar{\nu}$ is only of order $10^{-4}-10^{-5}$. Numerical results for various single particle spectra and their dependence on the pion momentum cutoff schemes are presented in a series of figures, as are the model independent ratios for differential rates of $D$ and $D^{*}$. We also study the parity-violation effects on the decay rates for different polarization states of the $D^{*}$.
\end{abstract}




\section{Introduction}

Flavor and spin symmetry of heavy quarks $[1,2]$ and chiral symmetry of light quarks together impose strong restrictions on the semileptonic decays of a $\bar{B}$ meson [3-6] such as $\bar{B} \rightarrow D \pi \ell \bar{\nu}$ and $\bar{B} \rightarrow D^{*} \pi \ell \bar{\nu}$. Heavy quark symmetry predicts that the weak vertex contains only a universal Isgur-Wise function with an overall factor calculable in QCD. Chiral symmetry, on the other hand, requires one unknown coupling constant to describe soft pion emissions from any ground-state heavy meson at low energies. Thus, the semileptonic decays with a soft pion are completely determined by the Isgur-Wise function measured in $\bar{B} \rightarrow D^{*} \ell \bar{\nu}$ and the coupling constant that describes the strong decay $D^{*} \rightarrow D \pi$. The Feynman diagrams for the decays to be studied in this work are shown in Figs. 1 and 2. The matrix elements for these decays are explicitly given in [3]. In this paper, we explore the implications in detail. Furthermore, since the unknowns appear as an overall factor of the decay matrix elements, many of the ratios of the differential spectra are free of any adjustable parameter. Some of these ratios are presented.

There is another motivation to study the semileptonic decays of the $\bar{B}$ meson with emission of additional pions. The 1992 Particle Data Group (PDG) [7] gives

$$
\begin{gathered}
B\left(B^{0} \rightarrow D^{-} \ell^{+} \nu\right)=(1.8 \pm 0.5) \%, \\
B\left(B^{0} \rightarrow D^{*-} \ell^{+} \nu\right)=(4.9 \pm 0.8) \%, \\
B\left(B^{+} \rightarrow \bar{D}^{0} \ell^{+} \nu\right)=(1.6 \pm 0.7) \%, \\
B\left(B^{+} \rightarrow \bar{D}^{* 0} \ell^{+} \nu\right)=(4.6 \pm 1.0) \%, \\
B\left(B \rightarrow e^{ \pm} \nu_{e} \text { hadrons }\right)=(10.7 \pm 0.5) \%,
\end{gathered}
$$

where $\ell$ indicates $e$ or $\mu$ mode (not sum over modes), and the charge of $B$ is not determined in the last branching ratio (1.5). Clearly, beside the $D \ell \bar{\nu}$ and $D^{*} \ell \bar{\nu}$ modes, there exist other important semileptonic decays of the $\bar{B}$ meson. There are indications from ARGUS [8] and CLEO [9] that $\bar{B} \rightarrow D^{* *} \ell \bar{\nu}$ gives a significant contribution. It is still interesting to ask how large the branching ratios for $\bar{B} \rightarrow D \pi \ell \bar{\nu}$ and $\bar{B} \rightarrow D^{*} \pi \ell \bar{\nu}$ are. It turns out that the branching ratio for $\bar{B} \rightarrow D \pi \ell \bar{\nu}$ can be quite significant, perhaps as large as $1 \%$, while the branching ratio for $\bar{B} \rightarrow D^{*} \pi \ell \bar{\nu}$ is much smaller, of order $10^{-4}$ to $10^{-5}$.

We would like to comment on our work in relation to other recent studies on similar subjects $[10,11,12]$. Lee, $\mathrm{Lu}$ and Wise [10] have extended the earlier formalisms for the $K_{\ell 4}$ and $D_{\ell 4}$ decays [13] to $D \rightarrow K \pi \bar{\ell} \nu, D \rightarrow \pi \pi \bar{\ell} \nu, B \rightarrow \pi \pi \bar{\ell} \nu$, and $\bar{B} \rightarrow D \pi \ell \bar{\nu}$. For the last decay, Lee et al. [10] have considered the predictions of chiral perturbation theory and heavy quark symmetry. They have calculated the decay rate in the region where chiral perturbation theory is expected to be valid. Kramer and Palmer [12] have discussed the decay $\bar{B} \rightarrow D^{*} \ell \bar{\nu} \rightarrow D \pi \ell \bar{\nu}$ in the resonant region and calculated the rate and angular correlation parameters in the framework of chiral and heavy quark symmetry. Lee [11] has generalized the analysis of [10] to the decay $\bar{B} \rightarrow D^{*} \pi \ell \bar{\nu}$ and has considered only the decay rates with the $D^{*}$ polarizations summed over. In our work, starting with the amplitudes

given in [3], we derive explicit formulae for the differential decay rates of both $\bar{B} \rightarrow D \pi \ell \bar{\nu}$ 
and $\bar{B} \rightarrow D^{*} \pi \ell \bar{\nu}$. Single particle energy spectra for $D$ or $D^{*}$, the electron and the pion are evaluated numerically; their dependence on the pion momentum cutoff schemes is studied. In the case of $\bar{B} \rightarrow D \pi \ell \bar{\nu}$, the $D^{*}$ pole dominates the amplitude, and the rates for the $D \pi$ system in the resonant and nonresonant regions (to be defined in Section V) are sensitive to the total decay width of $D^{*}$. Although the charged $D^{* \pm}$ decay almost exclusively to $D \pi$, the neutral $D^{* 0}$ has a substantial radiative decay contribution $[14,15]$. Consequently, the widths of $D^{*}$ are not simply related to the $D^{*} D \pi$ coupling constant. With our theoretical results for the $D^{*} D \pi$ coupling constant and the total widths of $D^{* \pm}$ and $D^{* 0}$ [14], we are able to predict the decay rates for $\bar{B} \rightarrow D^{*} \ell \bar{\nu}$ and $\bar{B} \rightarrow(D \pi)_{\text {nonres }} \ell \bar{\nu}$. The definitions of resonanting $D \pi$ (to be identified with $D^{*}$ ) and nonresonanting $D \pi$ are given in Section V. Our results for $\bar{B} \rightarrow D^{*} \ell \bar{\nu}$ for both charged and neutral $\bar{B}$ mesons agree with the available data (1.2) and (1.4). Our approach differs from those of [10] and [12]. In [10] it is suggested that the decay rates for $\bar{B} \rightarrow D \pi \ell \bar{\nu}$ can be used to fix the $D^{*} D \pi$ coupling constant. This is possible in principle provided that the $D \pi$ system is sufficiently far away from the $D^{*}$ resonance so that its rate is calculable without knowing the $D^{*}$ widths. But then the rates are rather small. On the other hand, the authors of [12] proposed that the decays $\bar{B} \rightarrow(D \pi)_{\text {res }} \ell \bar{\nu}$ can be used to determine the $D^{*} D \pi$ coupling constant. As we have pointed out earlier, this will require information on other decay modes of $D^{*}$.

It is not straightforward to compare our numerical results with those presented in $[10,11,12]$. As already mentioned, Lee, Lu and Wise [10] restrict themselves to the kinematic region away from the $D^{*}$ pole in $\bar{B} \rightarrow D \pi+e \bar{\nu}$. However, we include the region close to the resonance in our calculation. Lee [11] does not work out the total rates for $\bar{B} \rightarrow D^{*} \pi+e \bar{\nu}$, and none of our single particle spectra correspond to his tabulated differential rates. A crude estimate indicates that our total rate for $\bar{B} \rightarrow D^{*} \pi+e \bar{\nu}$ would have agreed with his within about a factor of two if the same Isgur-Wise function and value of the $D^{*} D \pi$ coupling constant were used. Kramer and Palmer [12] did not specify which charged modes they considered. Our rates for $\bar{B} \rightarrow D \pi+e \bar{\nu}$ in the resonant $D \pi$ region agree with theirs within about a factor of two.

In the case of $\bar{B} \rightarrow D^{*} \pi \ell \bar{\nu}$, the single particle spectra are calculated for polarized $D^{*}$. The underlying $V-A$ interaction of the quarks makes the spectra polarization dependent. Instead of describing all these results in words, we choose to present them in a series of figures.

The paper is organized as follows. In Section II we review the general kinematics of the four-body semileptonic decays. In Section III we make use of the matrix elements given in [3] to calculate the differential decay rates for $\bar{B} \rightarrow D \pi \ell \bar{\nu}$. The same analysis is carried out for $\bar{B} \rightarrow D^{*} \pi \ell \bar{\nu}$ in Section IV, with special attention given to the polarization of $D^{*}$. The results are discussed in Section V. We present the single particle spectra, the semileptonic rates versus the $D^{*}$ width, etc., in a series of figures. The connection between different Lorentz frames is considered in Appendix A. In Appendix B we derive a linear relation between the semileptonic decay rate and the inverse of $D^{*}$ width and discuss some of its implications. Some preliminary results have been reported earlier by two of us [16]. 


\section{Kinematics}

In this section we will review the kinematics of the decays

$$
\begin{aligned}
& \bar{B} \rightarrow D+\pi+\ell \bar{\nu}, \\
& \bar{B} \rightarrow D^{*}+\pi+\ell \bar{\nu} .
\end{aligned}
$$

General kinematics for such processes has been studied by several authors before [10-13, 17]. We will pay special attention to the new features for the latter where the polarization of $D^{*}$ is involved. It is well known that there are five independent kinematic variables for these processes if the spin of the initial state is zero or is not observed. Let the momentum of the $\bar{B}$ meson, $D$ (or $D^{*}$ ), the pion, the charged lepton, and the neutrino be $P_{B}, p, q, p_{\ell}$ and $p_{\nu}$, respectively. For the five variables we follow earlier authors' convention and pick

$s_{M}=(p+q)^{2}$

$s_{L}=\left(p_{\ell}+p_{\nu}\right)^{2}$,

$\theta=$ the angle between $\vec{p}$ in the $D\left(D^{*}\right) \pi$ rest frame and the line of the flight of the $D\left(D^{*}\right) \pi$ system in the $\bar{B}$ meson's rest frame,

$\theta_{\ell}=$ the angle between $\overrightarrow{p_{\ell}}$ in the $\ell \bar{\nu}$ rest frame and the line of flight of the $\ell \bar{\nu}$ system in the $\bar{B}$ meson's rest frame,

$\phi=$ the angle between the normals to the planes defined in the $\bar{B}$ meson's rest frame by the momenta of the $D\left(D^{*}\right) \pi$ pair and the $\ell \bar{\nu}$ pair, respectively. The sense of $\phi$ is from the $D\left(D^{*}\right) \pi$ plane to the $\ell \bar{\nu}$ plane.

These variables are depicted in Fig. 3. In the same figure, we also display the three orthonormal vectors associated with the 3 -momentum of $D^{*}$ in the $D^{*} \pi$ rest-frame. They will be useful for describing the polarization states of $D^{*}$. The positive $z$-axis is along the line of flight of the $D\left(D^{*}\right) \pi$ system in the $\bar{B}$ meson's rest frame; the $x$-axis is in the $D\left(D^{*}\right) \pi$ plane. The lepton mass will be neglected. We now form the combinations,

$$
\begin{gathered}
P=p+q, \quad Q=p-q, \\
L=p_{\ell}+p_{\nu}, \quad N=p_{\ell}-p_{\nu},
\end{gathered}
$$

and find

$$
\begin{gathered}
P \cdot L=\frac{1}{2}\left(m_{B}^{2}-s_{M}-s_{L}\right), \\
L \cdot N=0, \\
P \cdot Q=m^{2}-m_{\pi}^{2}, \\
Q^{2}=2\left(m^{2}+m_{\pi}^{2}\right)-s_{M}, \\
N^{2}=-s_{L},
\end{gathered}
$$


where $m^{2}=m_{D}^{2}$ or $m_{D^{*}}^{2}$ as the case may be.

Three frames of reference are of particular interest: the $B$-frame in which the $\bar{B}$ meson is at rest, the $M$-frame which is the center-of-mass frame of the $D\left(D^{*}\right) \pi$ system, and the $L$-frame which is the center-of-mass frame of the lepton pair. To construct some of the Lorentz invariants, it is often necessary to specify the individual components of various fourvectors in one of these coordinate systems. This information is provided in Appendix A. In particular, we find

$$
\begin{gathered}
Q \cdot N=\frac{\left(\frac{m^{2}-m_{\pi}^{2}}{s_{M}}\right) X \cos \theta_{\ell}+\beta(P \cdot L) \cos \theta \cos \theta_{\ell}}{-\sqrt{s_{M} s_{L}} \beta \sin \theta \sin \theta_{\ell} \cos \phi,} \\
\sigma \equiv \epsilon_{\mu \nu \lambda \kappa} Q^{\mu} P^{\nu} N^{\lambda} L^{\kappa}=-\sqrt{s_{M} s_{L}} \beta X \sin \theta \sin \theta_{\ell} \sin \phi \\
P_{B} \cdot p=\frac{1}{2}\left[\frac{s_{M}+m^{2}-m_{\pi}^{2}}{2 s_{M}}\left(m_{B}^{2}+s_{M}-s_{L}\right)+X \beta \cos \theta\right], \\
P_{B} \cdot q=\frac{1}{2}\left[\frac{s_{M}+m_{\pi}^{2}-m^{2}}{2 s_{M}}\left(m_{B}^{2}+s_{M}-s_{L}\right)-X \beta \cos \theta\right], \\
P_{B} \cdot p_{\ell}=\frac{1}{2}\left[\frac{1}{2}\left(m_{B}^{2}+s_{L}-s_{M}\right)+X \cos \theta_{\ell}\right],
\end{gathered}
$$

where $X$ and $\beta$ are defined in Appendix A, and our convention is $\epsilon_{0123}=1$.

In the laboratory frame (the $B$-frame), the above relations become

$$
\begin{aligned}
& P_{B} \cdot p=m_{B} E_{p} \\
& P_{B} \cdot q=m_{B} E_{q} \\
& P_{B} \cdot p_{\ell}=m_{B} E_{\ell}
\end{aligned}
$$

The four-body phase space element is given by

$$
\begin{aligned}
d(P S)= & \frac{d^{3} p}{(2 \pi)^{3} 2 E_{p}} \frac{d^{3} q}{(2 \pi)^{3} 2 E_{q}} \frac{d^{3} p_{\ell}}{(2 \pi)^{3} 2 E_{\ell}} \frac{d^{3} p_{\nu}}{(2 \pi)^{3} 2 E_{\nu}} \\
& \times(2 \pi)^{4} \delta^{4}\left(P_{B}-p-q-p_{\ell}-p_{\nu}\right) .
\end{aligned}
$$

To reduce the above expression to a five-dimensional integral, we insert the factors

$$
\begin{array}{ll}
1=\int \frac{d^{3} P}{2 E_{P}} d s_{M} \delta^{4}(P-p-q), & E_{P}=\sqrt{\vec{P}^{2}+s_{M}}, \\
1=\int \frac{d^{3} L}{2 E_{L}} d s_{L} \delta^{4}\left(L-p_{\ell}-p_{\nu}\right), & E_{L}=\sqrt{\vec{L}^{2}+s_{L}}
\end{array}
$$

then

$$
d(P S)=d s_{M} d s_{L} \frac{1}{(2 \pi)^{8}} I_{M} I_{L} I_{B}
$$


where

$$
\begin{aligned}
I_{M} & =\int \frac{d^{3} p}{2 E_{p}} \frac{d^{3} q}{2 E_{q}} \delta^{4}(P-p-q) \\
& =\frac{\pi}{4} \beta d \cos \theta, \\
I_{L} & =\int \frac{d^{3} p_{\ell}}{2 E_{\ell}} \frac{d^{3} p_{\nu}}{2 E_{\nu}} \delta^{4}\left(L-p_{\ell}-p_{\nu}\right) \\
& =\frac{1}{8} d \cos \theta_{\ell} d \phi, \\
I_{B} & =\int \frac{d^{3} P}{2 E_{P}} \frac{d^{3} L}{2 E_{L}} \delta^{4}\left(P_{B}-P-L\right) \\
& =\pi \frac{X}{m_{B}^{2}} \cdot
\end{aligned}
$$

Finally, we obtain the desired result

$$
d(P S)=\frac{1}{2(4 \pi)^{6} m_{B}^{2}} X \beta d s_{M} d s_{L} d \cos \theta d \cos \theta_{\ell} d \phi
$$

The region of integration is specified by

$$
\begin{gathered}
0<s_{L}<\left(m_{B}-\sqrt{s_{M}}\right)^{2} \\
\left(m+m_{\pi}\right)^{2}<s_{M}<m_{B}^{2} \\
0<\theta, \theta_{\ell}<\pi, \\
0<\phi<2 \pi .
\end{gathered}
$$

In the next two sections we will consider the single particle energy spectra in the laboratory frame (the $B$-frame). For this purpose we can make use of the relations (2.4) and (2.5) to change variables from $s_{M}$ and $s_{L}$ to $E_{p}$ and $E_{\ell}$ or $E_{q}$ and $E_{\ell}$. For example,

$$
d s_{M} d s_{L}=\left[\frac{\partial\left(E_{p}, E_{\ell}\right)}{\partial\left(s_{M}, s_{L}\right)}\right]^{-1} d E_{p} d E_{\ell}
$$

The Jacobian in (2.12) can be computed from (2.4) and (2.5). Other expressions for the fourbody phase space element which were useful in doing numerical calculations and verifying results are found in [18]. 


\section{The Semileptonic Decay $\bar{B} \rightarrow D+\pi+\ell \bar{\nu}$}

The general formalism for this type of decay has been worked out by several authors [3,10-12]. The hadronic matrix element for this process contains four form factors. We will not repeat the analysis here. In an earlier work [3] we have shown that the combined heavy quark symmetry and chiral symmetry requires only a single form factor to describe the decay, provided the emitted pion is soft.

The essential results are summarized below. The effective Lagrangian for semileptonic weak decays is given by

$$
\mathcal{L}_{\text {eff }}=\frac{G_{F}}{\sqrt{2}} J^{\mu} j_{\mu}
$$

where $G_{F}$ is the Fermi coupling constant, $J_{\mu}$ the charged hadronic weak current and $j_{\mu}$ the lepton's charged weak current. To be specific, let us consider $\bar{B}^{0} \rightarrow D^{+}+\pi^{0}+e^{-} \bar{\nu}_{e}$. We have followed the convention that a $\bar{B}$ meson contains a $b$ quark, while a $D$ meson contains a $c$ quark. The matrix element is given by

$$
M_{f i}=\frac{G_{F}}{\sqrt{2}} V_{c b}\left\langle\pi^{0}(q) D^{+}(p)\left|J_{\mu}^{c b}\right| \bar{B}^{0}\left(P_{B}\right)\right\rangle \bar{u}\left(p_{\ell}\right) \gamma^{\mu}\left(1-\gamma_{5}\right) v\left(p_{\nu}\right)
$$

where $V_{c b}$ is the CKM matrix element [19] for $b \rightarrow c$ transitions. We will write

$$
\left\langle\pi^{0}(q) D^{+}(p)\left|J_{\mu}^{c b}\right| \bar{B}^{0}\left(P_{B}\right)\right\rangle=\frac{i f}{2 f_{\pi}} \sqrt{m_{B} m_{D}} C_{c b} \xi H_{\mu},
$$

where the pion decay constant $f_{\pi}=93 \mathrm{MeV}, f$ is the $D^{*} D \pi$ coupling constant, $\xi$ is the universal Isgur-Wise function normalized to

$$
\xi\left(v \cdot v^{\prime}\right)=1 \quad \text { at } \quad v=v^{\prime},
$$

and $C_{c b}$ is the QCD correction factor

$$
\begin{gathered}
C_{c b}\left(v \cdot v^{\prime}\right)=\left[\frac{\alpha_{s}\left(m_{b}\right)}{\alpha_{s}\left(m_{c}\right)}\right]^{-\frac{6}{25}}\left[\frac{\alpha_{s}\left(m_{c}\right)}{\alpha_{s}(\mu)}\right]^{a_{L}\left(v \cdot v^{\prime}\right)}, \\
a_{L}(w)=\frac{8}{27}\left[\frac{w}{\sqrt{w^{2}-1}} \ln \left(w+\sqrt{w^{2}-1}\right)-1\right] .
\end{gathered}
$$

The quantity $H_{\mu}$ can be extracted from the result in [3]; it is given by

$$
H_{\mu}=w_{1} v_{\mu}+w_{2} v_{\mu}^{\prime}+r q_{\mu}+i h \epsilon_{\mu \nu \lambda \kappa} q^{\nu} v^{\prime \lambda} v^{\kappa}
$$


where the four-velocities $v$ and $v^{\prime}$ are defined by

$$
P_{B}=m_{B} v \quad, \quad p=m_{D} v^{\prime} .
$$

The form factors $w_{1,2}, r$ and $h$ are explicitly known in the soft pion limit:

$$
\begin{gathered}
w_{1}=\frac{q \cdot\left(v+v^{\prime}\right)}{2 v \cdot q+2 \Delta_{B}}, \\
w_{2}=-\frac{q \cdot\left(v+v^{\prime}\right)}{2 v^{\prime} \cdot q-2 \Delta_{D}}, \\
r=-\left(1+v \cdot v^{\prime}\right)\left[\frac{1}{2 v \cdot q+2 \Delta_{B}}-\frac{1}{2 v^{\prime} \cdot q-2 \Delta_{D}}\right] \\
h=\frac{1}{2 v \cdot q+2 \Delta_{B}}-\frac{1}{2 v^{\prime} \cdot q-2 \Delta_{D}} \\
\Delta_{B} \equiv m_{B^{*}}-m_{B} \quad, \quad \Delta_{D} \equiv m_{D^{*}}-m_{D} .
\end{gathered}
$$

In the numerical calculations, we have incorporated the finite width of $D^{*}, \Gamma_{D^{*}}$, to properly handle the $D^{*}$ resonance by making the replacement:

$$
\frac{1}{2 v^{\prime} \cdot q-2 \Delta_{D}} \rightarrow \frac{m_{D^{*}}}{(p+q)^{2}-m_{D^{*}}^{2}+i m_{D^{*}} \Gamma_{D^{*}}}
$$

The differential decay rate is then

$$
\begin{aligned}
d \Gamma\left(\bar{B}^{0} \rightarrow D^{+}+\pi^{0}+e^{-} \bar{\nu}_{\ell}\right) & =\frac{1}{2 m_{B}}\left|M_{f i}\right|^{2} d(P S) \\
& =\frac{G_{F}^{2} m_{D}}{8 m_{B}^{2}(4 \pi)^{6}}\left(\frac{f}{f_{\pi}} C_{c b} \xi\right)^{2}\left|V_{c b}\right|^{2}\left(\frac{1}{4} H_{\mu} H_{\nu}^{*} L^{\mu \nu}\right) \\
& \times \beta X d s_{M} d s_{L} d \cos \theta d \cos \theta_{\ell} d \phi
\end{aligned}
$$

where the lepton tensor $L_{\mu \nu}$ is given by

$$
L_{\mu \nu}=4\left(L_{\mu} L_{\nu}-N_{\mu} N_{\nu}-s_{L} g_{\mu \nu}-i \epsilon_{\mu \nu \lambda \kappa} L^{\lambda} N^{\kappa}\right)
$$

For a charged pion in the final state, the above expression (3.10) for $d \Gamma$ has to be multiplied by 2 due to isospin. A straightforward but tedious calculation gives 


$$
\begin{aligned}
& \frac{1}{4} H_{\mu} H_{\nu}^{*} L^{\mu \nu}=\left|w_{1} v \cdot L+w_{2} v^{\prime} \cdot L+r q \cdot L\right|^{2} \\
& -\left|w_{1} v \cdot N+w_{2} v^{\prime} \cdot N+r q \cdot N-i \frac{h}{2 m_{B} m_{D}} \sigma\right|^{2} \\
& -s_{L}\left\{\left|w_{1}\right|^{2}+\left(w_{1} w_{2}^{*}+w_{1}^{*} w_{2}\right) v \cdot v^{\prime}+\left(w_{1} r^{*}+w_{1}^{*} r\right) v \cdot q\right. \\
& +\left|w_{2}\right|^{2}+\left(w_{2} r^{*}+w_{2}^{*} r\right) v^{\prime} \cdot q+|r|^{2} m_{\pi}^{2}+ \\
& \left.+|h|^{2}\left[m_{\pi}^{2}\left(\left(v \cdot v^{\prime}\right)^{2}-1\right)-2(v \cdot q)\left(v^{\prime} \cdot q\right)\left(v \cdot v^{\prime}\right)+(q \cdot v)^{2}+\left(q \cdot v^{\prime}\right)^{2}\right]\right\} \\
& -i\left\{\frac{1}{2 m_{B} m_{D}}\left(w_{1} w_{2}^{*}-w_{1}^{*} w_{2}\right)-\frac{1}{2 m_{B}}\left(w_{1} r^{*}-w_{1}^{*} r\right)-\frac{1}{2 m_{D}}\left(w_{2} r^{*}-w_{2}^{*} r\right)\right\} \sigma \\
& +\left(h w_{1}^{*}+h^{*} w_{1}\right)\left[-(v \cdot q)\left(L \cdot v^{\prime}\right)(N \cdot v)-\left(v \cdot v^{\prime}\right)(L \cdot v)(N \cdot q)\right. \\
& \left.-(L \cdot q)\left(N \cdot v^{\prime}\right)+\left(v \cdot v^{\prime}\right)(L \cdot q)(N \cdot v)+\left(L \cdot v^{\prime}\right)(N \cdot q)+(v \cdot q)(L \cdot v)\left(N \cdot v^{\prime}\right)\right] \\
& +\left(h w_{2}^{*}+h^{*} w_{2}\right)\left[-\left(v^{\prime} \cdot q\right)\left(L \cdot v^{\prime}\right)(N \cdot v)-(L \cdot v)(N \cdot q)-\left(v \cdot v^{\prime}\right)(L \cdot q)\left(N \cdot v^{\prime}\right)\right. \\
& \left.+(L \cdot q)(N \cdot v)+\left(v \cdot v^{\prime}\right)\left(L \cdot v^{\prime}\right)(N \cdot q)+\left(v^{\prime} \cdot q\right)(L \cdot v)\left(N \cdot v^{\prime}\right)\right] \\
& +\left(h r^{*}+h^{*} r\right)\left[-m_{\pi}^{2}\left(L \cdot v^{\prime}\right)(N \cdot v)-\left(q \cdot v^{\prime}\right)(L \cdot v)(N \cdot q)\right. \\
& -(q \cdot v)(L \cdot q)\left(N \cdot v^{\prime}\right)+\left(q \cdot v^{\prime}\right)(L \cdot q)(N \cdot v)+(q \cdot v)\left(L \cdot v^{\prime}\right)(N \cdot q) \\
& \left.+m_{\pi}^{2}(L \cdot v)\left(N \cdot v^{\prime}\right)\right] .
\end{aligned}
$$

where $\sigma$ is the pseudoscalar defined by (2.4b). In deriving (3.12) we have also made use of the relations

$$
\begin{gathered}
\epsilon_{\mu \nu \lambda \kappa} N^{\mu} q^{\nu} v^{\prime \lambda} v^{\kappa}=-\frac{1}{2 m_{B} m_{D}} \sigma, \\
\epsilon_{\mu \nu \lambda \kappa} L^{\mu} N^{\nu} v^{\lambda} v^{\prime \kappa}=\frac{1}{2 m_{B} m_{D}} \sigma \\
\epsilon_{\mu \nu \lambda \kappa} v^{\prime \mu} q^{\nu} L^{\lambda} N^{\kappa}=-\frac{1}{2 m_{D}} \sigma \\
\epsilon_{\mu \nu \lambda \kappa} v^{\mu} q^{\nu} L^{\lambda} N^{\kappa}=-\frac{1}{2 m_{B}} \sigma
\end{gathered}
$$

With the help of $(2.4),(2.5)$ and $(2.12)$, we can work out the single-particle spectra $\frac{d \Gamma}{d E_{D}}, \frac{d \Gamma}{d E_{\pi}}$ and $\frac{d \Gamma}{d E_{\ell}}$. They will be discussed in Section $\mathrm{V}$. 
IV. The Semileptonic Decay $\bar{B} \rightarrow D^{*}+\pi+\ell \bar{\nu}$

The kinematics for this decay is very similar to the one discussed in previous sections. The new feature here is the polarization of the vector meson $D^{*}$ which we will exploit in our study.

In analogy with (3.3) for $\bar{B} \rightarrow D \pi \ell \bar{\nu}$ we define

$$
\left\langle D^{*+}(p) \pi^{0}(q)\left|J_{\mu}^{c b}\right| \bar{B}^{0}\left(P_{B}\right)\right\rangle=-\frac{i}{2} \sqrt{m_{B} m_{D^{*}}} \frac{f}{f_{\pi}} C_{c b} \xi H_{\mu}^{\prime},
$$

where an extra minus sign is introduced here compared with (3.3) due to the scalar products involving the polarization vector $\varepsilon_{\mu}$ of $D^{*}$. Heavy quark symmetry and chiral dynamics have a definite prediction for $H_{\mu}^{\prime}$ when the emitted pion is soft. In this limit we find from [3]

$$
\begin{aligned}
H_{\mu}^{\prime} & =a_{1} v_{\mu}+a_{2} v_{\mu}^{\prime}+a_{3} q_{\mu}+a_{4} \varepsilon_{\mu}^{*} \\
& +i \epsilon_{\mu \nu \lambda \kappa} \varepsilon^{* \nu}\left(b_{1} q^{\lambda} v^{\prime \kappa}+b_{2} q^{\lambda} v^{\kappa}+b_{3} v^{\lambda} v^{\prime \kappa}\right),
\end{aligned}
$$

where

$$
P_{B}=m_{B} v, \quad p=m_{D^{*}} v^{\prime},
$$

and

$$
\begin{aligned}
& a_{1}=-\left[\frac{1}{-2 v \cdot q-2 \Delta_{B}}+\frac{1}{2 v^{\prime} \cdot q+2 \Delta_{D}}\right]\left(\varepsilon^{*} \cdot q\right), \\
& a_{2}=\frac{1}{-2 v \cdot q-2 \Delta_{B}}\left[\left(\varepsilon^{*} \cdot v\right)(v \cdot q)-\varepsilon^{*} \cdot q\right] \\
& -\frac{1}{2 v^{\prime} \cdot q}\left[i \epsilon_{\rho \sigma \lambda \kappa} q^{\rho} \varepsilon^{* \sigma} v^{\prime \lambda} v^{\kappa}+\left(q \cdot v^{\prime}\right)\left(\varepsilon^{*} \cdot v\right)\right] \\
& -\frac{1}{2 v^{\prime} \cdot q+2 \Delta_{D}}\left(\varepsilon^{*} \cdot q\right) \text {, } \\
& a_{3}=\left[\frac{1}{-2 v \cdot q-2 \Delta_{B}}+\frac{1}{2 v^{\prime} \cdot q}\right]\left(\varepsilon^{*} \cdot v\right), \\
& \begin{aligned}
a_{4}= & \frac{1}{-2 v \cdot q-2 \Delta_{B}}\left[\left(q \cdot v^{\prime}\right)-\left(v \cdot v^{\prime}\right)(v \cdot q)\right] \\
& -\frac{1}{2 v^{\prime} \cdot q}\left[(q \cdot v)-\left(v \cdot v^{\prime}\right)\left(v^{\prime} \cdot q\right)\right],
\end{aligned} \\
& b_{1}=\frac{1}{-2 v \cdot q-2 \Delta_{B}}+\frac{1}{2 v^{\prime} \cdot q}\left(1+v \cdot v^{\prime}\right), \\
& b_{2}=\frac{1}{-2 v \cdot q-2 \Delta_{B}},
\end{aligned}
$$




$$
b_{3}=\frac{-(q \cdot v)}{-2 v \cdot q-2 \Delta_{B}} .
$$

In our numerical calculations for this process we will not employ the full propagator (3.9) as we do for the decay $\bar{B} \rightarrow D \pi \ell \bar{\nu}$, since none of the intermediate states can become real here.

The absolute value squared of the matrix element involves

$$
\begin{aligned}
\frac{1}{4} H_{\mu}^{\prime} H_{\nu}^{\prime *} L^{\mu \nu}= & \left|H^{\prime} \cdot L\right|^{2}-\left|H^{\prime} \cdot N\right|^{2}-s_{L} H^{\prime} \cdot H^{\prime *} \\
& -i \epsilon^{\mu \nu \lambda \kappa} H_{\mu}^{\prime} H_{\nu}^{\prime *} L_{\lambda} N_{\kappa},
\end{aligned}
$$

where the lepton tensor $L_{\mu \nu}$ is the same as the one given by (3.11). Each term in (4.5) is straightforward to compute, though it is tedious sometimes.

To begin with, we introduce the quantities

$$
\begin{gathered}
\sigma_{1}=\epsilon_{\mu \nu \lambda \kappa} N^{\mu} \varepsilon^{* \nu} q^{\lambda} v^{\prime \kappa}, \\
\sigma_{2}=\epsilon_{\mu \nu \lambda \kappa} N^{\mu} \varepsilon^{* \nu} q^{\lambda} v^{\kappa}, \\
\sigma_{3}=\epsilon_{\mu \nu \lambda \kappa} N^{\mu} \varepsilon^{* \nu} v^{\lambda} v^{\prime \kappa}, \\
\sigma_{4}=\epsilon_{\mu \nu \lambda \kappa} v^{\mu} \varepsilon^{* \nu} q^{\lambda} v^{\prime \kappa} .
\end{gathered}
$$

and

$$
\begin{gathered}
\sigma_{1}^{\prime}=\epsilon_{\mu \nu \lambda \kappa} \varepsilon^{* \mu} \varepsilon^{\nu} q^{\lambda} v^{\prime \kappa}, \\
\sigma_{2}^{\prime}=\epsilon_{\mu \nu \lambda \kappa} \varepsilon^{* \mu} \varepsilon^{\nu} q^{\lambda} v^{\kappa}, \\
\sigma_{3}^{\prime}=\epsilon_{\mu \nu \lambda \kappa} \varepsilon^{* \mu} \varepsilon^{\nu} v^{\lambda} v^{\prime \kappa}, \\
\sigma_{4}^{\prime}=\epsilon_{\mu \nu \lambda \kappa} \varepsilon^{* \mu} \varepsilon^{\nu} L^{\lambda} N^{\kappa} .
\end{gathered}
$$

Then, we find

$$
\begin{aligned}
& H^{\prime} \cdot L=a_{1} v \cdot L+a_{2} v^{\prime} \cdot L+a_{3} q \cdot L+a_{4} \varepsilon^{*} \cdot L \\
& +i \sigma_{4}\left(m_{B} b_{1}+m_{D^{*}} b_{2}+b_{3}\right) \\
& H^{\prime} \cdot N=a_{1} v \cdot N+a_{2} v^{\prime} \cdot N+a_{3} q \cdot N+a_{4} \varepsilon^{*} \cdot N \\
& +i\left(\sigma_{1} b_{1}+\sigma_{2} b_{2}+\sigma_{3} b_{3}\right) \\
& H^{\prime} \cdot H^{\prime *}=T_{a}+T_{a b}+T_{b} \\
& T_{a}=\left|a_{1}\right|^{2}+\left|a_{2}\right|^{2}+\left|a_{3}\right|^{2} m_{\pi}^{2}-\left|a_{4}\right|^{2}+\left(a_{1} a_{2}^{*}+a_{1}^{*} a_{2}\right) v \cdot v^{\prime} \\
& +\left(a_{1} a_{3}^{*}+a_{1}^{*} a_{3}\right) v \cdot q+\left(a_{2} a_{3}^{*}+a_{2}^{*} a_{3}\right) v^{\prime} \cdot q \\
& +a_{1} a_{4}^{*} \varepsilon \cdot v+a_{1}^{*} a_{4} \varepsilon^{*} \cdot v+a_{3} a_{4}^{*} \varepsilon \cdot q+a_{3}^{*} a_{4} \varepsilon^{*} \cdot q \text {, }
\end{aligned}
$$




$$
\begin{aligned}
T_{a b}= & -i a_{1} b_{1}^{*} \sigma_{4}^{*}+i a_{1}^{*} b_{1} \sigma_{4}+i a_{2} b_{2}^{*} \sigma_{4}^{*}-i a_{2}^{*} b_{2} \sigma_{4} \\
& +i a_{3} b_{3}^{*} \sigma_{4}^{*}-i a_{3}^{*} b_{3} \sigma_{4} \\
& -i\left(a_{4} b_{1}^{*}+a_{4}^{*} b_{1}\right) \sigma_{1}^{\prime}-i\left(a_{4} b_{2}^{*}+a_{4}^{*} b_{2}\right) \sigma_{2}^{\prime} \\
& -i\left(a_{4} b_{3}^{*}+a_{4}^{*} b_{3}\right) \sigma_{3}^{\prime}
\end{aligned}
$$

$$
\begin{aligned}
T_{b}= & \left|b_{1}\right|^{2}\left[m_{\pi}^{2}+|\varepsilon \cdot q|^{2}-\left(q \cdot v^{\prime}\right)^{2}\right] \\
& +\left|b_{2}\right|^{2}\left[m_{\pi}^{2}-\left(\varepsilon^{*} \cdot q\right)(\varepsilon \cdot v)(q \cdot v)-\left(\varepsilon^{*} \cdot v\right)(\varepsilon \cdot q)(q \cdot v)+|\varepsilon \cdot q|^{2}\right. \\
& \left.+|\varepsilon \cdot v|^{2} m_{\pi}^{2}-(q \cdot v)^{2}\right] \\
& +\left|b_{3}\right|^{2}\left[1+|\varepsilon \cdot v|^{2}-\left(v \cdot v^{\prime}\right)^{2}\right] \\
& +b_{1} b_{2}^{*}\left[m_{\pi}^{2}\left(v \cdot v^{\prime}\right)-\left(\varepsilon^{*} \cdot v\right)(\varepsilon \cdot q)\left(v^{\prime} \cdot q\right)+\left|\varepsilon^{*} \cdot q\right|^{2}\left(v \cdot v^{\prime}\right)-(q \cdot v)\left(q \cdot v^{\prime}\right)\right] \\
& +b_{1}^{*} b_{2}\left[m_{\pi}^{2}\left(v \cdot v^{\prime}\right)-(\varepsilon \cdot v)\left(\varepsilon^{*} \cdot q\right)\left(v^{\prime} \cdot q\right)+\left|\varepsilon^{*} \cdot q\right|^{2}\left(v \cdot v^{\prime}\right)-(q \cdot v)\left(q \cdot v^{\prime}\right)\right] \\
& +b_{2} b_{3}^{*}\left[(q \cdot v)\left(v \cdot v^{\prime}\right)-|\varepsilon \cdot v|^{2}\left(q \cdot v^{\prime}\right)+\left(\varepsilon^{*} \cdot v\right)(\varepsilon \cdot q)\left(v \cdot v^{\prime}\right)-\left(q \cdot v^{\prime}\right)\right] \\
& +b_{2}^{*} b_{3}\left[(q \cdot v)\left(v \cdot v^{\prime}\right)-|\varepsilon \cdot v|^{2}\left(q \cdot v^{\prime}\right)+(\varepsilon \cdot v)\left(\varepsilon^{*} \cdot q\right)\left(v \cdot v^{\prime}\right)-\left(q \cdot v^{\prime}\right)\right] \\
& +b_{3} b_{1}^{*}\left[(q \cdot v)+\left(\varepsilon^{*} \cdot q\right)(\varepsilon \cdot v)-\left(q \cdot v^{\prime}\right)\left(v \cdot v^{\prime}\right)\right] \\
& +b_{3}^{*} b_{1}\left[(q \cdot v)+(\varepsilon \cdot q)\left(\varepsilon^{*} \cdot v\right)-\left(q \cdot v^{\prime}\right)\left(v \cdot v^{\prime}\right)\right] .
\end{aligned}
$$

Similarly, we write

$$
i \epsilon^{\mu \nu \lambda \kappa} H_{\mu}^{\prime} H_{\nu}^{\prime *} L_{\lambda} N_{\kappa}=R_{a}+R_{a b}+R_{b}
$$

where

$$
\begin{aligned}
R_{a}= & i\left|a_{4}\right|^{2} \sigma_{4}^{\prime}+\operatorname{Im}\left\{\sigma\left(-\frac{1}{m_{B} m_{D^{*}}} a_{1} a_{2}^{*}+\frac{1}{m_{B}} a_{1} a_{3}^{*}+\frac{1}{m_{D^{*}}} a_{2} a_{3}^{*}\right)\right. \\
& -2 \sigma_{1}^{*}\left(a_{2} a_{4}^{*}-m_{D^{*}} a_{3} a_{4}^{*}\right)-2 \sigma_{2}^{*}\left(m_{B} a_{3} a_{4}^{*}+a_{1} a_{4}^{*}\right) \\
& \left.+2 \sigma_{3}^{*}\left(m_{D^{*}} a_{1} a_{4}^{*}+m_{B} a_{2} a_{4}^{*}\right)\right\}
\end{aligned}
$$




$$
\begin{aligned}
R_{a b}= & 2 \operatorname{Re}\left\{a _ { 1 } ^ { * } b _ { 1 } \left[\left(v \cdot \varepsilon^{*}\right)(L \cdot q)\left(N \cdot v^{\prime}\right)+\left(N \cdot \varepsilon^{*}\right)(v \cdot q)\left(L \cdot v^{\prime}\right)+\left(L \cdot \varepsilon^{*}\right)(N \cdot q)\left(v \cdot v^{\prime}\right)\right.\right. \\
& \left.-\left(L \cdot \varepsilon^{*}\right)(v \cdot q)\left(N \cdot v^{\prime}\right)-\left(N \cdot \varepsilon^{*}\right)(L \cdot q)\left(v \cdot v^{\prime}\right)-\left(v \cdot \varepsilon^{*}\right)(N \cdot q)\left(L \cdot v^{\prime}\right)\right] \\
& +a_{1}^{*} b_{2}\left[\left(v \cdot \varepsilon^{*}\right)(L \cdot q)(N \cdot v)+\left(N \cdot \varepsilon^{*}\right)(v \cdot q)(L \cdot v)+\left(L \cdot \varepsilon^{*}\right)(N \cdot q)\right. \\
& \left.-\left(L \cdot \varepsilon^{*}\right)(v \cdot q)(N \cdot v)-\left(N \cdot \varepsilon^{*}\right)(L \cdot q)-\left(v \cdot \varepsilon^{*}\right)(N \cdot q)(L \cdot v)\right] \\
& +a_{1}^{*} b_{3}\left[\left(v \cdot \varepsilon^{*}\right)(L \cdot v)\left(N \cdot v^{\prime}\right)+\left(N \cdot \varepsilon^{*}\right)\left(L \cdot v^{\prime}\right)+\left(L \cdot \varepsilon^{*}\right)(N \cdot v)\left(v \cdot v^{\prime}\right)\right. \\
& \left.-\left(L \cdot \varepsilon^{*}\right)\left(N \cdot v^{\prime}\right)-\left(N \cdot \varepsilon^{*}\right)(L \cdot v)\left(v \cdot v^{\prime}\right)-\left(v \cdot \varepsilon^{*}\right)(N \cdot v)\left(L \cdot v^{\prime}\right)\right] \\
& +a_{2}^{*} b_{1}\left[\left(N \cdot \varepsilon^{*}\right)\left(v^{\prime} \cdot q\right)\left(L \cdot v^{\prime}\right)+\left(L \cdot \varepsilon^{*}\right)(N \cdot q)\right. \\
& \left.-\left(L \cdot \varepsilon^{*}\right)\left(v^{\prime} \cdot q\right)\left(N \cdot v^{\prime}\right)-\left(N \cdot \varepsilon^{*}\right)(L \cdot q)\right] \\
& +a_{2}^{*} b_{2}\left[\left(N \cdot \varepsilon^{*}\right)\left(v^{\prime} \cdot q\right)(L \cdot v)+\left(L \cdot \varepsilon^{*}\right)(N \cdot q)\left(v \cdot v^{\prime}\right)-\left(L \cdot \varepsilon^{*}\right)\left(v^{\prime} \cdot q\right)(N \cdot v)\right. \\
& \left.-\left(N \cdot \varepsilon^{*}\right)(L \cdot q)\left(v \cdot v^{\prime}\right)\right] \\
& +a_{2}^{*} b_{3}\left[\left(N \cdot \varepsilon^{*}\right)\left(v \cdot v^{\prime}\right)\left(L \cdot v^{\prime}\right)+\left(L \cdot \varepsilon^{*}\right)(N \cdot v)-\left(L \cdot \varepsilon^{*}\right)\left(v \cdot v^{\prime}\right)\left(N \cdot v^{\prime}\right)\right. \\
& \left.-\left(N \cdot \varepsilon^{*}\right)(L \cdot v)\right] \\
& +a_{3}^{*} b_{1}\left[\left(q \cdot \varepsilon^{*}\right)(L \cdot q)\left(N \cdot v^{\prime}\right)+\left(N \cdot \varepsilon^{*}\right) m_{\pi}^{2}\left(L \cdot v^{\prime}\right)+\left(L \cdot \varepsilon^{*}\right)(N \cdot q)\left(q \cdot v^{\prime}\right)\right. \\
& \left.-\left(L \cdot \varepsilon^{*}\right) m_{\pi}^{2}\left(N \cdot v^{\prime}\right)-\left(N \cdot \varepsilon^{*}\right)(L \cdot q)\left(q \cdot v^{\prime}\right)-\left(q \cdot \varepsilon^{*}\right)(N \cdot q)\left(L \cdot v^{\prime}\right)\right] \\
& +a_{3}^{*} b_{2}\left[\left(q \cdot \varepsilon^{*}\right)(L \cdot q)(N \cdot v)+\left(N \cdot \varepsilon^{*}\right) m_{\pi}^{2}(L \cdot v)+\left(L \cdot \varepsilon^{*}\right)(N \cdot q)(q \cdot v)\right. \\
& \left.-\left(L \cdot \varepsilon^{*}\right) m_{\pi}^{2}(N \cdot v)-\left(N \cdot \varepsilon^{*}\right)(L \cdot q)(q \cdot v)-\left(q \cdot \varepsilon^{*}\right)(N \cdot q)(L \cdot v)\right] \\
& +a_{3}^{*} b_{3}\left[\left(q \cdot \varepsilon^{*}\right)(L \cdot v)\left(N \cdot v^{\prime}\right)+\left(N \cdot \varepsilon^{*}\right)(q \cdot v)\left(L \cdot v^{\prime}\right)+\left(L \cdot \varepsilon^{*}\right)(N \cdot v)\left(q \cdot v^{\prime}\right)\right. \\
& \left.-\left(L \cdot \varepsilon^{*}\right)(q \cdot v)\left(N \cdot v^{\prime}\right)-\left(N \cdot \varepsilon^{*}\right)(L \cdot v)\left(q \cdot v^{\prime}\right)-\left(q \cdot \varepsilon^{*}\right)(N \cdot v)\left(L \cdot v^{\prime}\right)\right] \\
& +a_{4}^{*} b_{1}\left[-(L \cdot q)\left(N \cdot v^{\prime}\right)+\left(N \cdot \varepsilon^{*}\right)(\varepsilon \cdot q)\left(L \cdot v^{\prime}\right)-\left(L \cdot \varepsilon^{*}\right)(\varepsilon \cdot q)\left(N \cdot v^{\prime}\right)\right. \\
& \left.+(N \cdot q)\left(L \cdot v^{\prime}\right)\right] \\
& +a_{4}^{*} b_{2}\left[-(L \cdot q)(N \cdot v)+\left(N \cdot \varepsilon^{*}\right)(\varepsilon \cdot q)(L \cdot v)+\left(L \cdot \varepsilon^{*}\right)(N \cdot q)(\varepsilon \cdot v)\right. \\
& \left.-\left(L \cdot \varepsilon^{*}\right)(\varepsilon \cdot q)(N \cdot v)-\left(N \cdot \varepsilon^{*}\right)(L \cdot q)(\varepsilon \cdot v)+(N \cdot q)(L \cdot v)\right] \\
& +a_{4}^{*} b_{3}\left[-(L \cdot v)\left(N \cdot v^{\prime}\right)+\left(N \cdot \varepsilon^{*}\right)(\varepsilon \cdot v)\left(L \cdot v^{\prime}\right)-\left(L \cdot \varepsilon^{*}\right)(\varepsilon \cdot v)\left(N \cdot v^{\prime}\right)\right. \\
& \left.\left.+(N \cdot v)\left(L \cdot v^{\prime}\right)\right]\right\}, \\
& \left(L^{\prime}\right)(N)
\end{aligned}
$$

and

$$
\begin{aligned}
R_{b}= & -\operatorname{Im}\left\{\left[\left(L \cdot v^{\prime}\right)(N \cdot q)-(L \cdot q)\left(N \cdot v^{\prime}\right)\right]\left(\left|b_{1}\right|^{2} \sigma_{1}^{\prime}+b_{2}^{*} b_{1} \sigma_{2}^{\prime}+b_{3}^{*} b_{1} \sigma_{3}^{\prime}\right)\right. \\
& +[(L \cdot v)(N \cdot q)-(L \cdot q)(N \cdot v)]\left(\left|b_{2}\right|^{2} \sigma_{2}^{\prime}+b_{1}^{*} b_{2} \sigma_{1}^{\prime}+b_{3}^{*} b_{2} \sigma_{3}^{\prime}\right) \\
& +\left[\left(L \cdot v^{\prime}\right)(N \cdot v)-(L \cdot v)\left(N \cdot v^{\prime}\right)\right]\left(\left|b_{3}\right|^{2} \sigma_{3}^{\prime}+b_{2}^{*} b_{3} \sigma_{2}^{\prime}+b_{1}^{*} b_{3} \sigma_{1}^{\prime}\right) \\
& +\left[(L \cdot q)\left(N \cdot \varepsilon^{*}\right)-\left(L \cdot \varepsilon^{*}\right)(N \cdot q)\right]\left(b_{1}^{*} b_{2}-b_{2}^{*} b_{1}\right) \sigma_{4}^{*} \\
& +\left[\left(L \cdot \varepsilon^{*}\right)(N \cdot v)-(L \cdot v)\left(N \cdot \varepsilon^{*}\right)\right]\left(b_{2}^{*} b_{3}-b_{3}^{*} b_{2}\right) \sigma_{4}^{*} \\
& \left.+\left[\left(L \cdot v^{\prime}\right)\left(N \cdot \varepsilon^{*}\right)-\left(L \cdot \varepsilon^{*}\right)\left(N \cdot v^{\prime}\right)\right]\left(b_{3}^{*} b_{1}-b_{1}^{*} b_{3}\right) \sigma_{4}^{*}\right\}
\end{aligned}
$$

The polarization state of a massive vector meson is not a Lorentz invariant concept. A state with a definite polarization in one frame of reference will become a linear combination of states with different polarizations in another frame of reference. When the polarization of a vector meson is specified, we have to give the frame of reference in which it is defined. In our numerical calculations and results to be presented in the next section, we will employ states of $D^{*}$ with definite polarizations in the rest frame of the $\bar{B}$ meson (the $B$-frame). The polarization vectors in the $M$-frame and their Lorentz transforms in the $L$-frame are provided in Appendix A. Polarization vectors in the $B$-frame are not explicitly given, but they are not difficult to construct. 


\section{Results and Discussion}

In this section we make use of the results obtained in the last two sections to compute the single particle spectra for the charmed meson $\left(D\right.$ or $\left.D^{*}\right)$, the pion and the electron, and the total rates for $\bar{B} \rightarrow D+\pi+\ell \bar{\nu}$ and $\bar{B} \rightarrow D^{*}+\pi+\ell \bar{\nu}$. These results are presented in a series of figures. The energies in the single particle spectra of these figures are those measured in the rest frame of the $\bar{B}$ meson; the polarizations of $D^{*}$ are also specified in the $B$-frame. Since the validity of chiral symmetry demands the emitted pions be soft, we must impose cutoffs on the pion momenta in our calculation. It is not clear how soft a pion must be for chiral symmetry to work, nor is it obvious in which reference frame the pion has to be soft. For the problem at hand, there are two obvious frames: the rest frame of the $\bar{B}$ meson and the rest frame of $D\left(D^{*}\right)$ system. As the pion is soft, the center of mass frame of the $D\left(D^{*}\right) \pi$ system is approximately the same as the rest frame of the $D\left(D^{*}\right)$ meson. We refer to the latter as the $D$-frame or the $D^{*}$-frame as the case may be. Results are presented with the pion momentum cutoff in the $B$-frame, the $D$-frame, or both. We simply cut off the pion's 3-momentum at $100 \mathrm{MeV} / \mathrm{c}$ or $200 \mathrm{MeV} / \mathrm{c}$ in the appropriate frame of reference. Comparison among the plots for different cutoffs should give some idea of the sensitivity of our results to the different cutoff procedures. Generally speaking, the shapes of various spectra do not differ very much. The rate for $\bar{B} \rightarrow D \pi \ell \bar{\nu}$ does not change by much, but that for $\bar{B} \rightarrow D^{*} \pi \ell \bar{\nu}$ varies by almost an order of magnitude.

Throughout our calculations, we use the following values of the well-measured parameters $[7,20]$ :

$$
\begin{aligned}
& m_{B^{ \pm}}=5278.6 \mathrm{MeV}, \quad m_{B^{0}}=5278.7 \mathrm{MeV}, \quad m_{B^{*}}=5331.3 \mathrm{MeV}, \\
& m_{D^{0}}=1864.5 \mathrm{MeV}, \quad m_{D^{ \pm}}=1869.3 \mathrm{MeV}, \quad m_{D^{* \pm}}=2010.1 \mathrm{MeV}, \\
& m_{D^{* 0}}=2007.1 \mathrm{MeV}, \quad f_{\pi}=93.0 \mathrm{MeV}, \quad G_{F}=1.16637 \times 10^{-11} \mathrm{MeV}^{-2} .
\end{aligned}
$$

We also use

$$
f=-1.5, \quad \Gamma_{D^{*+}}=141 \mathrm{keV}, \quad \Gamma_{D^{* 0}}=102 \mathrm{keV} .
$$

In (5.2), the value for the fundamental coupling constant $f$ is the one from the quark model given in our earlier work [3], and the $D^{*}$ widths are our prediction [14]. We also used other values for $\Gamma_{D^{*}}$ as shown in Figs. 7-10. As for the Isgur-Wise form factor, we use the one given by Burdman [21]

$$
\xi(y)=1-\rho^{2}(y-1)+c(y-1)^{2} .
$$

with

$$
\rho=1.08 \pm 0.10, \quad c=0.62 \pm 0.15 .
$$

In his fit Burdman found

$$
\left|V_{c b}\right|=0.041 \pm 0.005 \pm 0.002,
$$


which is somewhat smaller than the values obtained by several experimental analyses [22]. But it is not easy to compare (5.5) with other analyses since Burdman's fit includes QCD corrections. As a result, $\xi(y)$ given by (5.3) is only applicable to $\bar{B} \rightarrow D\left(D^{*}\right)$ decays.

We now consider some details of the decays $\bar{B}^{0} \rightarrow D+\pi+\ell \bar{\nu}$. Since the intermediate $D^{*}$ can be on its mass shell, results for this decay rate are separated into two categories: resonant and nonresonant. The resonant part is defined as those events with the invariant mass of $D \pi$ satisfying [23]:

$$
\left|m(D \pi)-m_{D^{*}}\right|<3 \Gamma_{D^{*}} .
$$

All others are nonresonant. For comparison with experiment, the resonant part is identified as $\bar{B}^{0} \rightarrow D^{*} \ell \bar{\nu}$ followed by the decay of the $D^{*}$ to the specific $D \pi$ state, while the nonresonant part is identified as $\bar{B}^{0} \rightarrow D \pi \ell \bar{\nu}$. We have found that the contribution from the $D^{*}$ pole dominates both the resonant and nonresonant decays. Since the most important Feynman diagram is Fig. 1b, where the pion is emitted in a transition from $D^{*}$ to $D$, it is most reasonable to cut the pion's three momentum off in the $D$-frame. We see from Figs. 4-6 that the shapes do not change much between the single-particle spectra in the resonant region and their counterparts in the nonresonant region. However, the rates in the resonant region are larger than those in the nonresonant region by a factor of 7 . When the cutoff increases from $100 \mathrm{MeV}$ to $200 \mathrm{MeV}$, the nonresonant rate increases by about $15 \%$. The pion momentum cutoff of $100 \mathrm{MeV}$ or $200 \mathrm{MeV}$ in the $D$-frame has no effect on the resonant contribution, since the pion momentum in this frame is only about $40 \mathrm{MeV}$. We have investigated the sensitivity to $\Gamma_{D^{*}}$ of the shapes of the single particle spectra. They hardly change as $\Gamma_{D^{*}}$ varies from $0.1 \mathrm{MeV}$ to $1 \mathrm{MeV}$.

To estimate the branching ratios of $\bar{B}^{0} \rightarrow(D \pi)_{\text {res }}+e^{-} \bar{\nu}_{e}$ and $\bar{B}^{0} \rightarrow(D \pi)_{\text {nonres }}+e^{-} \bar{\nu}_{e}$, we first convert the mean lifetime of $\bar{B}$ mesons [7]

$$
\tau_{B}=(12.9 \pm 0.5) \times 10^{-13} s,
$$

to a total decay width of

$$
\Gamma_{B}=\frac{\hbar}{\tau}=(0.51 \pm 0.02) \times 10^{-9} \mathrm{MeV} .
$$

The width $\Gamma_{D^{*}}$ also affects the total decay rates for $\bar{B}^{0} \rightarrow(D \pi)_{\text {res }}+e^{-} \bar{\nu}_{e}$ and $\bar{B}^{0} \rightarrow$ $(D \pi)_{\text {nonres }}+e^{-} \bar{\nu}_{e}$. The dependence of the integrated rates on $\Gamma_{D^{*}}$ is displayed in Figs. 7-10. We have fixed the value of $f$ in the amplitudes by (5.2), but have treated $\Gamma_{D^{*}}$ as a free parameter in the $D^{*}$ propagator (3.9). In this way, a linear relationship between the integrated rates and $1 / \Gamma_{D^{*}}$ is expected theoretically. The details are presented in Appendix B. We notice that as $\Gamma_{D^{*}}$ varies from $0.1 \mathrm{MeV}$ to $1 \mathrm{MeV}, \Gamma\left[\bar{B}^{0} \rightarrow\left(D^{+} \pi^{0}\right)_{\text {res }}+e^{-} \bar{\nu}_{e}\right]$ decreases from about $1.2 \times 10^{-11} \mathrm{MeV}$ to $1.2 \times 10^{-12} \mathrm{MeV}$ for $q_{\max }=100 \mathrm{MeV}$ or $q_{\max }=200 \mathrm{MeV}$. For $q_{\max }=100 \mathrm{MeV}$, the corresponding change for the rate $\Gamma\left[\bar{B}^{0} \rightarrow\left(D^{+} \pi^{0}\right)_{\text {nonres }}+e^{-} \bar{\nu}_{e}\right]$ is from $1.5 \times 10^{-12} \mathrm{MeV}$ to $2.2 \times 10^{-13} \mathrm{MeV}$. For $q_{\max }=200 \mathrm{MeV}$, the corresponding change 
in the nonresonant rate is from $1.6 \times 10^{-12} \mathrm{MeV}$ to $3.7 \times 10^{-13} \mathrm{MeV}$. Similar variations are also found in the rates for $B^{-} \rightarrow D^{0} \pi^{0} e^{-} \bar{\nu}_{e}$ with respect to changes of $\Gamma_{D^{*}}$ and the pion momentum cutoffs. Because $m_{D^{* 0}}<m_{D^{+}}+m_{\pi^{-}}$, the rates for $B^{-} \rightarrow\left(D^{+} \pi^{-}\right)_{\text {res }} e^{-} \bar{\nu}_{e}$ are completely negligible. The rates for $B^{-} \rightarrow\left(D^{+} \pi^{-}\right)_{\text {nonres }} e^{-} \bar{\nu}_{e}$ are nearly independent of $\Gamma_{D^{*}}$. See appendix $\mathrm{B}$ for more discussion on $B^{-} \rightarrow D^{+} \pi^{-} e^{-} \bar{\nu}_{e}$.

From Figs. 7-10, we find the decay rates with a neutral pion

$$
\begin{gathered}
\Gamma\left[\bar{B}^{0} \rightarrow\left(D^{+} \pi^{0}\right)_{\mathrm{res}}+e^{-} \bar{\nu}_{e}\right]=8.50 \times 10^{-12} \mathrm{MeV}, \\
\Gamma\left[\bar{B}^{0} \rightarrow\left(D^{+} \pi^{0}\right)_{\mathrm{nonres}}+e^{-} \bar{\nu}_{e}\right]=1.09 \times 10^{-12} \mathrm{MeV}, \\
\Gamma\left[B^{-} \rightarrow\left(D^{0} \pi^{0}\right)_{\mathrm{res}}+e^{-} \bar{\nu}_{e}\right]=1.78 \times 10^{-11} \mathrm{MeV}, \\
\Gamma\left[B^{-} \rightarrow\left(D^{0} \pi^{0}\right)_{\text {nonres }}+e^{-} \bar{\nu}_{e}\right]=2.16 \times 10^{-12} \mathrm{MeV} .
\end{gathered}
$$

The corresponding branching ratios are

$$
\begin{gathered}
B\left[\bar{B}^{0} \rightarrow\left(D^{+} \pi^{0}\right)_{\mathrm{res}}+e^{-} \bar{\nu}_{e}\right]=1.67 \%, \\
B\left[\bar{B}^{0} \rightarrow\left(D^{+} \pi^{0}\right)_{\mathrm{nonres}}+e^{-} \bar{\nu}_{e}\right]=0.21 \%, \\
B\left[B^{-} \rightarrow\left(D^{0} \pi^{0}\right)_{\mathrm{res}}+e^{-} \bar{\nu}_{e}\right]=3.49 \%, \\
B\left[B^{-} \rightarrow\left(D^{0} \pi^{0}\right)_{\mathrm{nonres}}+e^{-} \bar{\nu}_{e}\right]=0.42 \% .
\end{gathered}
$$

If we identify the $D \pi^{\prime}$ s in the resonant region with the $D^{*}$, Eq. (5.10a) is the combined branching ratio for $\bar{B}^{0} \rightarrow D^{*+} e^{-} \bar{\nu}_{e}$ and $D^{*+} \rightarrow D^{+} \pi^{0}$. The decay $D^{*+} \rightarrow D^{+} \pi^{0}$ is predicted to have a branching ratio of $31.2 \%$ [14], thus

$$
\begin{aligned}
B\left[\bar{B}^{0} \rightarrow D^{*+}+e^{-} \bar{\nu}_{e}\right] & =\frac{B\left[\bar{B}^{0} \rightarrow\left(D^{+} \pi^{0}\right)_{\mathrm{res}}+e^{-} \bar{\nu}_{e}\right]}{B\left[D^{*+} \rightarrow D^{+} \pi^{0}\right]} \\
& =5.35 \% .
\end{aligned}
$$

The agreement between (5.11) and the data (1.2) is very good. Encouraged by this success, we would like to relate $(5.10 \mathrm{c})$ to the branching ratio of $B^{-} \rightarrow D^{* 0} e^{-} \bar{\nu}_{e}$. To do this we notice that $D^{* 0} \rightarrow D^{+} \pi^{-}$is kinematically forbidden, and $D^{* 0}$ has a substantial radiative decay. Using the branching ratio of $66.7 \%$ for $D^{* 0} \rightarrow D^{0} \pi^{0}$ [14], we find

$$
B\left[B^{-} \rightarrow D^{* 0}+e^{-} \bar{\nu}_{e}\right]=5.23 \%,
$$

which agrees with the data (1.4). The success of the predictions (5.11) and (5.12) represents a triumph for heavy quark symmetry; it is independent of the chiral symmetry of light quarks. For the nonresonant $D \pi$ final states, we can read off from Figs. 8 and 10 the contribution from processes with a charged pion. Combining this with (5.9) and (5.10), we find

$$
\begin{aligned}
& B\left[\bar{B}^{0} \rightarrow(D \pi)_{\text {nonres }}^{+}+e^{-} \bar{\nu}_{e}\right]=0.68 \%, \\
& B\left[B^{-} \rightarrow(D \pi)_{\text {nonres }}^{0}+e^{-} \bar{\nu}_{e}\right]=0.45 \% .
\end{aligned}
$$

We notice in passing that isospin symmetry is reasonably good for $\bar{B}^{0}$ decays but not so for $B^{-}$decays, as a consequence of the fact that $m_{D^{* 0}}<m_{D^{+}}+m_{\pi^{-}}$. So far, the results quoted above are for $q_{\max }=100 \mathrm{MeV}$ in the $D^{*}$ frame. For $q_{\max }=200 \mathrm{MeV}$, we obtain

$$
B\left[\bar{B}^{0} \rightarrow(D \pi)_{\text {nonres }}^{+}+e^{-} \bar{\nu}_{e}\right]=0.77 \%,
$$


Table I. Integrated rates of $\bar{B}^{0} \rightarrow D^{*+} \pi^{0} e^{-} \bar{\nu}_{e}$ with different pion momentum cutoff.

We also list the branching ratios and the percentage contributions from each polarization of $D^{*}$ in the $B$-frame. The longitudinal, left-handed and right-handed polarizations are labelled $L_{0}, L$ and $R$, respectively.

\begin{tabular}{lcccccc}
\hline \hline $\begin{array}{l}\text { cutoff } \\
\text { frame }\end{array}$ & $\begin{array}{c}\text { pion momentum } \\
\text { cutoff }(\mathrm{MeV})\end{array}$ & rate $(\mathrm{MeV})$ & $\begin{array}{c}\text { branching } \\
\text { ratio }\end{array}$ & $L_{0}(\%)$ & $L(\%)$ & $R(\%)$ \\
\hline$B$ & 100 & $3.20 \times 10^{-15}$ & $0.63 \times 10^{-5}$ & 26 & 57 & 17 \\
$B$ & 200 & $1.84 \times 10^{-14}$ & $0.36 \times 10^{-4}$ & 30 & 52 & 18 \\
$B$ and $D^{*}$ & 100 & $9.62 \times 10^{-16}$ & $0.19 \times 10^{-5}$ & 27 & 54 & 19 \\
$B$ and $D^{*}$ & 200 & $1.04 \times 10^{-14}$ & $0.20 \times 10^{-4}$ & 33 & 48 & 19 \\
\hline \hline
\end{tabular}

$$
B\left[B^{-} \rightarrow(D \pi)_{\text {nonres }}^{0}+e^{-} \bar{\nu}_{e}\right]=0.53 \%
$$

As mentioned in the Introduction, there appears to be a deficit between the branching ratio for the inclusive semileptonic decays $B \rightarrow e^{ \pm} \nu_{e}$ thadrons and the sum of the two exclusive channels $B^{0} \rightarrow D^{-} \ell^{+} \nu$ and $B^{0} \rightarrow D^{*-} \ell^{+} \nu$. Our study shows that the nonresonant decay $\bar{B}^{0} \rightarrow D \pi \ell \bar{\nu}$ can have a substantial branching ratio, although not enough to account for the difference. Nevertheless, it is measurable and is interesting in its own right.

Before we leave the subject of $\bar{B} \rightarrow(D \pi)_{\text {nonres }} \ell \bar{\nu}$, we would like to repeat a comment made in Appendix B. The results for nonresonant contributions (5.9b), (5.9d), (5.13) and (5.14) are very sensitive to the definition (5.6) for resonant contributions. To compare our predictions with future experiments, we must bear this point in mind. Of course, the sum of the resonant and nonresonant contributions is independent of this arbitrary division into resonant and nonresonant parts.

We now turn to the decay $\bar{B}^{0} \rightarrow D^{*+} \pi^{0} e^{-} \bar{\nu}_{e}$. The results are shown in Figs. 11-16. The overall rates are smaller than $\bar{B}^{0} \rightarrow D \pi \ell \bar{\nu}$ by two or three orders of magnitude. For this process, we have imposed the pion momentum cutoff in the $B$-frame or in both the $B$-frame and the $D^{*}$-frame. We see that different cutoffs give significantly different rates. In addition to the figures, integrated rates for $\bar{B}^{0} \rightarrow D^{*+} \pi^{0} e^{-} \bar{\nu}_{e}$ are given in Table I.

The polarization of the vector meson $D^{*}$ is a new feature of this decay. It can be exploited to study the nature of weak interaction dynamics. In Figs. 11-16, we show the single particle spectra for each polarization of $D^{*}$ in the $B$-frame. In all cases, contributions from the lefthanded and longitudinal polarizations dominate that from the right-handed polarization. This can be simply understood as a result of the $V-A$ coupling of the quarks to the $W^{ \pm}$ bosons. The charmed quark produced by the $\bar{B}$ decay is predominantly left-handed. The helicity of this charmed quark will not be affected by the creation of the soft pion by the light quark interactions. A simple reflection will show that a left-handed charmed quark can only lead to a vector meson $D^{*}$ with a left-handed polarization or a longitudinal polarization. Since the charmed quark has a finite mass, there is some contamination from the right- 
handed component. This contamination should be small when the charmed quark (hence $\left.D^{*}\right)$ is energetic. This reasoning is indeed borne out by our calculations.

Our results so far rely on a specific fit to the Isgur-Wise form factor and a choice of the values of $f, V_{c b}$, etc. All these uncertainties will disappear if we take the ratio of the corresponding quantities at the same value of $v \cdot v^{\prime}$ in the decays $\bar{B} \rightarrow D \pi \ell \bar{\nu}$ and $\bar{B} \rightarrow D^{*} \pi \ell \bar{\nu}$. These ratios are the model independent predictions from the heavy quark symmetry and chiral symmetry. They are displayed in Figs. 17-20. We await the day when we will be able to compare these curves with experimental data.

To get a better idea of how the decay rates depend on the pion momentum cutoffs, we have extrapolated our results beyond the soft pion limit. In Figs. 21-23, we show the various decay rates as a function of the pion momentum cutoff in the $B$-frame, the $D$-frame, or both. Since $\bar{B} \rightarrow(D \pi)_{\text {nonres }} \ell \bar{\nu}$ is dominated by the $D^{*}$ pole, it is not very sensitive to a change in cutoff. Unfortunately, for $\bar{B} \rightarrow D^{*} \pi \ell \bar{\nu}$ the rates vary rapidly with the cutoff at low pion momenta. This exercise raises an important question: What constitutes a soft pion?

Finally, so far we have completely neglected the contribution from $\bar{B} \rightarrow D^{* *} \ell \bar{\nu}, D^{* *} \rightarrow$ $D^{*} \pi$. Theoretically, this is justified since $D^{* *}$ and $D^{*}$ are nondegenerate, so the amplitude vanishes in the soft pion limit. However, this contribution can be important in practice. It certainly deserves further study [24].

\section{Acknowledgments}

One of us (T.M.Y.) would like to express his deep appreciation of the hospitality extended to him by the Theory Group at the Institute of Physics, Academia Sinica, Taipei, Taiwan, ROC during his stay there where part of the work was done. H.Y.C. wishes to thank Prof. C. N. Yang and the Institute for Theoretical Physics at Stony Brook for their hospitality during his stay there for sabbatical leave. T.M.Y.'s work is supported in part by the National Science Foundation. This research is suppored in part by the National Science Council of ROC under Contract Nos. NSC82-0208-M001-001Y, NSC82-0208-M001-016, NSC82-0208M001-060 and NSC82-0208-M008-012. 
Table II. Components of various four-vectors in the center of mass frame of $D\left(D^{*}\right) \pi$ (the $M$-Frame)

\begin{tabular}{ccccc}
\hline \hline$a^{\mu}$ & $a^{x}$ & $a^{y}$ & $a^{z}$ & $a^{0}$ \\
\hline$p^{\mu}$ & $p \sin \theta$ & 0 & $p \cos \theta$ & $\frac{1}{2 \sqrt{s_{M}}}\left(s_{M}-m_{\pi}^{2}+m^{2}\right)$ \\
$L^{\mu}$ & 0 & 0 & $-L$ & $\frac{1}{\sqrt{s_{M}}} P \cdot L$ \\
$e_{1}^{\mu}$ & $\cos \theta$ & 0 & $-\sin \theta$ & 0 \\
$e_{2}^{\mu}$ & 0 & 1 & 0 & 0 \\
$e_{3}^{\mu}$ & $\frac{p^{0}}{m} \sin \theta$ & 0 & $\frac{p^{0}}{m} \cos \theta$ & $\frac{p}{m}$ \\
\hline \hline
\end{tabular}

\section{Appendix A}

In this appendix we present some of the properties of various four-momenta in the three coordinate systems mentioned in the text (the $B-, M-$, and $L$-frames). Many of the results can be found in the literature $[10,13,17]$.

In the center of mass system of $D\left(D^{*}\right) \pi$ (the $M$-frame), we have

$$
\begin{aligned}
p & =|\vec{p}|=|\vec{q}| \\
& =\frac{1}{2} \sqrt{s_{M}} \beta \\
L & =|\vec{L}|=\frac{X}{\sqrt{s_{M}}},
\end{aligned}
$$

where

$$
X=\sqrt{(P \cdot L)^{2}-s_{M} s_{L}}
$$

and

$$
\beta=\frac{1}{s_{M}}\left[s_{M}^{2}-2 s_{M}\left(m^{2}+m_{\pi}^{2}\right)+\left(m^{2}-m_{\pi}^{2}\right)^{2}\right]^{\frac{1}{2}} .
$$

In the $M$-frame, the components of the four-vectors $P^{\mu}$ and $L^{\mu}$ as well as the linear polarization vectors $e_{1,2,3}^{\mu}$ are given in Table II. The values of $|\vec{p}|$ and $P \cdot L$ are given by (A.1) and (2.3a), respectively. The three linear polarization vectors of $D^{*}$ in the $M$-frame as indicated in Fig. 3 are denoted by $e_{1}^{\mu}, e_{2}^{\mu}$, and $e_{3}^{\mu}$. Their components in the $M$-frame are listed in Table II.

Sometimes, we need to know the Lorentz transformations that connect the different frames of references. A simple calculation gives the parameters

$$
\beta_{M L}=\frac{X}{P \cdot L}
$$


Table III. Components of various four-vectors in the rest frame of the lepton pair (the $L$-Frame)

\begin{tabular}{lcccc}
\hline \hline$a^{\mu}$ & $a^{x}$ & $a^{y}$ & $a^{z}$ & $a^{0}$ \\
\hline$L^{\mu}$ & 0 & 0 & 0 & $\sqrt{s_{L}}$ \\
$P^{\mu}$ & 0 & 0 & $X / \sqrt{s_{L}}$ & $P \cdot L / \sqrt{s_{L}}$ \\
$Q^{\prime \mu}$ & $\sqrt{s_{M}} \beta \sin \theta$ & 0 & $P \cdot L \beta \cos \theta / \sqrt{s_{L}}$ & $X \beta \cos \theta / \sqrt{s_{L}}$ \\
$p_{\ell}^{\mu}$ & $\frac{1}{2} \sqrt{s_{L}} \sin \theta_{\ell} \cos \phi$ & $-\frac{1}{2} \sqrt{s_{L}} \sin \theta_{\ell} \sin \phi$ & $-\frac{1}{2} \sqrt{s_{L}} \cos \theta_{\ell}$ & $\frac{1}{2} \sqrt{s_{L}}$ \\
\hline \hline
\end{tabular}

$$
\beta_{M B}=\frac{X}{P \cdot L+s_{M}}
$$

If $a_{L}^{\mu}$ and $a_{M}^{\mu}$ are components of the same four-vector in the $L$-frame and the $M$-frame, respectively, they are related by the Lorentz transformations

$$
\begin{aligned}
& a_{L}^{z}=\gamma_{M L}\left(a_{M}^{z}+\beta_{M L} a_{M}^{0}\right), \\
& a_{L}^{0}=\gamma_{M L}\left(a_{M}^{0}+\beta_{M L} a_{M}^{z}\right)
\end{aligned}
$$

Similar equations hold using $\beta_{M B}$ to relate $a_{B}^{\mu}$ and $a_{M}^{\mu}$, the components of a vector in the $B$ and $M$ frames.

From $Q^{\mu}$ and $P^{\mu}$ we can construct another four-momentum which is orthogonal to $P^{\mu}$ :

$$
Q_{\mu}^{\prime}=Q_{\mu}-\frac{m^{2}-m_{\pi}^{2}}{s_{M}} P_{\mu}
$$

with

$$
Q^{\prime} \cdot P=0
$$

Now, we are ready to list the components of various four-vectors in different frames of reference. In the $L$-frame, the components of various four-vectors are listed in Table III.

The components of $P_{B}$ and $p_{\nu}$ can be inferred from the ones given above in the Tables. Furthermore, with the help of a Lorentz transformation we can determine the components of all the four-vectors in another coordinate system. 


\section{Appendix B}

In this appendix we derive a linear relation between the semileptonic decay rates with a soft pion emission and the inverse of the $D^{*}$ width. In what follows, the $D^{*} D \pi$ coupling constant $f$ which appears in the pion emission vertices is held fixed, while the width $\Gamma_{D^{*}}$ is considered to be a free parameter in the $D^{*}$ propagator.

We should emphasize that our numerical work does not make the approximation presented below. The purpose of this appendix is to understand the regularities exhibited in the numerical results of Figs. 7-10.

Consider the Feynman diagram with the $D^{*}$ pole contributing to $\bar{B} \rightarrow D \pi \ell \bar{\nu}$ (Fig. $1 \mathrm{~b}$ ). The matrix element can be written as

$$
M(\bar{B} \rightarrow D \pi \ell \bar{\nu})=\sum_{\lambda} M\left[D^{*}(\lambda) \rightarrow D \pi\right] \frac{1}{P^{2}-m^{2}+i m \Gamma_{D^{*}}} M\left[\bar{B} \rightarrow D^{*}(\lambda) \ell \bar{\nu}\right]
$$

where $\lambda$ denotes the polarization of $D^{*}$, and $P$ and $m$ are the 4-momentum and mass of $D^{*}$, respectively. For example,

$$
M\left[D^{*}(\lambda) \rightarrow D \pi^{a}\right]=u^{*}\left(D^{*}\right) \frac{1}{2} \tau^{a} u(D) \sqrt{m_{D} m} \frac{f}{f_{\pi}} \varepsilon(\lambda) \cdot q
$$

where $q$ is the pion momentum. The decay rate for $\bar{B} \rightarrow D \pi \ell \bar{\nu}$ due to the $D^{*}$ pole is

$$
\begin{aligned}
\Gamma_{\mathrm{D}^{*} \text { pole }}(\bar{B} \rightarrow D \pi \ell \bar{\nu}) & =\frac{1}{2 m_{B}} \frac{1}{2 \pi} \sum_{\lambda} \int d s_{M}\left|M\left[\bar{B} \rightarrow D^{*}(\lambda) \ell \bar{\nu}\right]\right|^{2} \\
& \times(2 \pi)^{4} \delta^{4}\left(P_{B}-p_{\ell}-p_{\nu}-P\right) \frac{d^{3} p_{\ell}}{(2 \pi)^{3} 2 E_{\ell}} \frac{d^{3} p_{\nu}}{(2 \pi)^{3} 2 E_{\nu}} \frac{d^{3} P}{(2 \pi)^{3} 2 E_{P}} \\
& \times \frac{1}{\left(s_{M}-m^{2}\right)^{2}+m^{2} \Gamma_{D^{*}}^{2}} \int\left|M\left[D^{*}(\lambda) \rightarrow D \pi\right]\right|^{2} \\
& \times(2 \pi)^{4} \delta^{4}(P-q-p) \frac{d^{3} p}{(2 \pi)^{3} 2 E_{p}} \frac{d^{3} q}{(2 \pi)^{3} 2 E_{q}} .
\end{aligned}
$$

This equation follows from inserting the factor

$$
1=\int d s_{M} \frac{d^{3} P}{2 E_{P}} \delta^{4}(P-q-p), \quad E_{P} \equiv \sqrt{\vec{P}^{2}+s_{M}},
$$

and the observation that one of the double sums over $D^{*}$ polarizations after squaring (B.1) is eliminated when we carry out the integration over the directions of $\vec{q}$. Making use of the standard formula for a decay width, we find

$$
\Gamma_{\mathrm{D}^{*} \text { pole }}(\bar{B} \rightarrow D \pi \ell \bar{\nu})=\frac{1}{\pi} \int d s_{M} \frac{\sqrt{s_{M}} \Gamma\left(\bar{B} \rightarrow D^{*} \ell \bar{\nu}, s_{M}\right) \Gamma\left(D^{*} \rightarrow D \pi, s_{M}\right)}{\left(s_{M}-m^{2}\right)^{2}+m^{2} \Gamma_{D^{*}}^{2}}
$$


To obtain (B.5), we have used the fact that the width of the decay $D^{*} \rightarrow D \pi$ is independent of the $D^{*}$ polarization. The argument $s_{M}$ appears in the numerator of the integrand of (B.5) because the widths are those appropriate for $D^{*}$ with a mass $\sqrt{s_{M}}$.

Let us introduce the new variables

$$
\begin{gathered}
s_{M}=m^{2}+x m \Gamma_{D^{*}}, \\
F\left(s_{M}\right)=\sqrt{s_{M}} \Gamma\left(\bar{B} \rightarrow D^{*} \ell \bar{\nu}, s_{M}\right) \Gamma\left(D^{*} \rightarrow D \pi, s_{M}\right) .
\end{gathered}
$$

Eq.(B.5) now becomes

$$
\Gamma_{\mathrm{D}^{*} \text { pole }}(\bar{B} \rightarrow D \pi \ell \bar{\nu})=\frac{1}{m \Gamma_{D^{*}}} \frac{1}{\pi} \int d x \frac{1}{x^{2}+1} F\left[m^{2}+x m \Gamma_{D^{*}}\right]
$$

When the width $\Gamma_{D^{*}}$ is small, the integrand can be expanded in a Taylor series. The leading term is

$$
\Gamma_{\mathrm{D}^{*} \text { pole }}(\bar{B} \rightarrow D \pi \ell \bar{\nu})=\frac{F\left(m^{2}\right)}{m \Gamma_{D^{*}}} \frac{1}{\pi} \int d x \frac{1}{x^{2}+1}+\cdots
$$

If the range of $s_{M}$ is not restricted, after the $x$-integration we obtain

$$
\Gamma_{\mathrm{D}^{*} \text { pole }}(\bar{B} \rightarrow D \pi \ell \bar{\nu})=\Gamma\left(\bar{B} \rightarrow D^{*} \ell \bar{\nu}\right) \frac{\Gamma\left(D^{*} \rightarrow D \pi\right)}{\Gamma_{D^{*}}}+\cdots,
$$

where (B.6b) has been used. This is a well-known result in the theory of resonances. In practice, experimental cuts are imposed on the range of $s_{M}$. Suppose the cut is

$$
\left|\sqrt{s_{M}}-m\right|<N \Gamma_{D^{*}}
$$

which corresponds to

$$
-2 N+N^{2} \frac{\Gamma_{D^{*}}}{m}<x<2 N+N^{2} \frac{\Gamma_{D^{*}}}{m}
$$

then the region (B.10) or (B.11) is the resonant contribution and outside this region is the nonresonant contribution. Finally,

$$
\begin{array}{r}
\Gamma\left[\bar{B} \rightarrow(D \pi)_{\mathrm{res}}+\ell \bar{\nu}\right]=\left(\frac{2}{\pi} \tan ^{-1} 2 N\right) \frac{1}{\Gamma_{D^{*}}} \cdot \frac{F\left(m^{2}\right)}{m}+A, \\
\Gamma\left[\bar{B} \rightarrow(D \pi)_{\text {nonres }}+\ell \bar{\nu}\right]=\left(1-\frac{2}{\pi} \tan ^{-1} 2 N\right) \frac{1}{\Gamma_{D^{*}}} \cdot \frac{F\left(m^{2}\right)}{m}+A^{\prime} .
\end{array}
$$

The constant terms $A$ and $A^{\prime}$ which are independent of $\Gamma_{D^{*}}$ may arise from the nonleading contributions of $\Gamma_{\mathrm{D}^{*} \text { pole }}$, the $B^{*}$-pole contributions, and the interference terms between the $B^{*}$-pole and $D^{*}$-pole contributions. These constants $A$ and $A^{\prime}$ are generally very different for resonant and nonresonant contributions. 
The definition (5.6) for resonant contributions corresponds to $N=3$ and for this choice we have

$$
\begin{gathered}
\Gamma\left[\bar{B} \rightarrow(D \pi)_{\mathrm{res}}+\ell \bar{\nu}\right]=0.895 \frac{1}{\Gamma_{D^{*}}} \cdot \frac{F\left(m^{2}\right)}{m}+A, \\
\Gamma\left[\bar{B} \rightarrow(D \pi)_{\mathrm{nonres}}+\ell \bar{\nu}\right]=0.105 \frac{1}{\Gamma_{D^{*}}} \cdot \frac{F\left(m^{2}\right)}{m}+A^{\prime} .
\end{gathered}
$$

These linear relations are confirmed by the numerical results shown in Figs. 7-10 for $\bar{B}^{0} \rightarrow$ $D^{+} \pi^{0} e^{-} \bar{\nu}_{e}, \bar{B}^{0} \rightarrow D^{0} \pi^{+} e^{-} \bar{\nu}_{e}$, and $B^{-} \rightarrow D^{0} \pi^{0} e^{-} \bar{\nu}_{e}$. The ratio of slopes of the two linear relations is

$$
\frac{(\text { slope })_{\text {res }}}{(\text { slope })_{\text {nonres }}}=\frac{0.895}{0.105}=8.52 \text {, }
$$

which agrees with the slopes in Figs. $7-10$, as it can be easily verified. The decays $B^{-} \rightarrow$ $D^{+} \pi^{-} e^{-} \bar{\nu}_{e}$ deserve special attention. First of all, $B^{-} \rightarrow\left(D^{+} \pi^{-}\right)_{\text {res }} e^{-} \bar{\nu}_{e}$ is kinematically forbidden if $\Gamma_{D^{* 0}} \leq 0.4 \mathrm{MeV}$, since $m\left(D^{+} \pi^{-}\right)$always falls outside the resonant condition (5.6). For $\Gamma_{D^{* 0}}$ between 0.4 and $1 \mathrm{MeV}$, the phase space is so restricted that the decay rates for $B^{-} \rightarrow\left(D^{+} \pi^{-}\right)_{\text {res }} e^{-} \bar{\nu}_{e}$ are completely negligible. Furthermore, the decay rate for $B^{-} \rightarrow\left(D^{+} \pi^{-}\right)_{\text {nonres }} e^{-} \bar{\nu}_{e}$ is found to be rather small and independent of $\Gamma_{D^{*}}$ as seen in Fig. 10. This behavior can be understood by the following considerations. We notice that the leading term in the approximation (B.8) vanishes since there is no phase space for the decay $D^{* 0} \rightarrow D^{+} \pi^{-}$(see the mass values given in (5.1)). In addition, from (5.1) we have

$$
P^{2}=(p+q)^{2} \geq\left(m_{D^{+}}+m_{\pi^{-}}\right)^{2}
$$

or

$$
P^{2}-m_{D^{* 0}}^{2} \geq 7500 \mathrm{MeV}^{2}
$$

as compared with

$$
\text { 200.7 } \mathrm{MeV}^{2} \leq m_{D^{* 0}} \Gamma_{D^{* 0}} \leq 2007 \mathrm{MeV}^{2},
$$

for $0.1 \mathrm{MeV} \leq \Gamma_{D^{* 0}} \leq 1 \mathrm{MeV}$. We conclude that in the denominator of the $D^{*}$ propagator of (B.1), the imaginary part is always small and hence it can be neglected. The result is therefore independent of $\Gamma_{D^{* 0}}$. Moreover, the denominator of the $D^{*}$ propagator is never very small and the phase space near its minimum (B.15) is very limited. As a consequence, the decay rate is substantially reduced.

The definition (5.6) for resonant contributions is reasonable, but somewhat arbitrary. However, the decay rate for resonant contributions is rather insensitive to the definition. As $N$ varies from $N=2$ to $N=\infty$, the slope in (B.13a) changes by $-5.7 \%$ and $+11.7 \%$, respectively. On the other hand, the nonresonant contributions are very sensitive to the experimental cuts.

Finally, Figs. 7-10 show that the straight lines for the resonant contributions for both charged and neutral $\bar{B}$ mesons pass through the origin. We conclude that $A \approx 0$. This is 
to be expected since the cut (5.6) gives rise to a very small phase space contributing to the constant $A$. 


\section{REFERENCES}

1. N. Isgur and M.B. Wise, Phys. Lett. B232, 113 (1989); Phys. Lett. B237, 527 (1990).

2. M.B. Voloshin and M.A. Shifman, Yad. Fiz. 45, 463 (1987) [Sov. J. Nucl. Phys. 45, $292(1987)]$.

3. T.M. Yan, H.Y. Cheng, C.Y. Cheung, G.L. Lin, Y.C. Lin, and H.L. Yu, Phys. Rev. D46, 1148 (1992). See also T.M. Yan, Chin. J. Phys. (Taipei) 30, 509 (1992).

4. M.B. Wise, Phys. Rev. D45, R2188 (1992).

5. G. Burdman and J. Donoghue, Phys. Lett. B280, 287 (1992).

6. P. Cho, Phys. Lett. B285, 145 (1992).

7. Particle Data Group, Phys. Rev. D45, S1 (1992).

8. The ARGUS Collaboration, H. Albrecht et al., Z. Phys. C57, 533 (1993).

9. The CLEO Collaboration, R. Fulton et al., Phys. Rev. 43, 651 (1992); The CLEO Collaboration, S. Hernandez et al., ibid D45, 2212 (1992).

10. Clarence L.Y. Lee, Ming Lu, and Mark B. Wise, Phys. Rev. D46, 5040 (1992).

11. Clarence L.Y. Lee, CALT-68-1841 (1992).

12. G. Kramer and W.F. Palmer, DESY 92-130 (1992); G. Köpp, G. Kramer, W. Palmer, and G. Schuler, Z. Phys. C48, 327 (1990).

13. A. Pais and S.B. Treiman, Phys. Rev. 168, 1858 (1968); see also, N. Cabibbo and A. Maksymowicz, Phys. Rev. 137, B438 (1965).

14. H.Y. Cheng, C.Y. Cheung, G.L. Lin, Y.C. Lin, T.M. Yan, and H.L. Yu, Phys. Rev. D47, 1030 (1993).

15. The CLEO Collaboration, F. Butler et al., Phys. Rev. Lett. 69, 2041 (1992).

16. G.L. Lin, invited talk delivered at the First Workshop on Particle Physics Phenomenology, Kenting, Taiwan, R.O.C., May 1992, IP-ASTP-16-92, to appear in Chin. J. Phys. (1993); H.Y. Cheng, IP-ASTP-18-92, to appear in Proceedings of the XXVI International Conference on High Energy Physics, Dallas, August 1992.

17. G.L.Kane, K. Stowe, and W.B. Rolnick, Nucl. Phys. B152, 390 (1979).

18. P. Nyborg and O. Skjeggestad, in Kinematics and Multiparticle Systems, Proceedings of the International School of Elementary Particle Physics, Herceg-Novi, Yugoslavia, 1965, edited by M. Nikolić. Pages 55-57 are especially useful for computing single particle energy spectra. 
19. N. Cabibbo, Phys. Rev. Lett. 10, 531 (1963); M. Kobayashi and K. Maskawa, Prog. Theor. Phys. 49, 652 (1993).

20. The value of the $B^{*}$ mass used here is from the 1990 edition of PDG [Phys. Lett. B239, $\mathrm{S} 1$ (1990)]. We have checked our results using its new value in $1992 \mathrm{PDG}, m_{B^{*}}=5324.6$ $\mathrm{MeV}$. The differences for $\bar{B} \rightarrow D \pi \ell \bar{\nu}$ are negligible and those for $\bar{B} \rightarrow D^{*} \pi \ell \bar{\nu}$ are less than $1 \%$.

21. G. Burdman, Phys. Lett. B284, 133 (1992).

22. For a review of the experimental status of $V_{c b}$, see Persis S. Drell and J. Richie Patterson, CLNS 92/1177, to appear in Proceedings of the XXVI International Conference on High Energy Physics, Dallas, August 1992.

23. We have benefited from conversations with Prof. P. Drell on the general question of how to define resonant and nonresonant contributions.

24. P. Bialas, K. Zalewski, and J.G. Körner, TPJU-19/92, MZ-TH/92-53 (1992); Ming Lu, M.B. Wise, and N. Isgur, Phys. Rev. D45, 1553 (1992). 


\section{Figure Captions}

Fig. 1 Feynman diagrams for $\bar{B} \rightarrow D \pi \ell \bar{\nu}$.

Fig. 2 Feynman diagrams for $\bar{B} \rightarrow D^{*} \pi \ell \bar{\nu}$.

Fig. 3 (a) General kinematics of $\bar{B} \rightarrow D\left(D^{*}\right) \pi \ell \bar{\nu}$. The dashed lines are the lines of flight of the $D\left(D^{*}\right) \pi$ pair and lepton pair in the rest frame of the $\bar{B}$ meson. Solid lines denote the line of flight of $D\left(D^{*}\right)$ and $\pi$ in the $M$-frame and that of the lepton and neutrino in the $L$-frame. The coordinates $x, y$, and $z$ are indicated, and the angles $\theta, \theta_{\ell}$ and $\phi$ are labeled. (b) The three linear polarization vectors of $D^{*}$ in the $M$-frame. The vector $\vec{e}_{1}$ is in X-Z plane, $\vec{e}_{2}$ is along the $\hat{y}$ axis and $\vec{e}_{3}$ is along $\vec{p}$.

Fig. 4 The energy spectra of the $D$ meson from $\bar{B}^{0} \rightarrow D^{+} \pi^{0} e^{-} \bar{\nu}_{e}$ in the resonant region (solid line) and in the nonresonant region (broken lines). Shown are effects of two pion momentum cutoffs (100 MeV and $200 \mathrm{MeV}$ ) in the $D$-frame on the nonresonant contributions. The bump at the high energy end is an artifact of the simple cutoff imposed on the pion. The resonant contribution is not affected by these pion momentum cutoffs. The spectrum is in units of $\mathrm{MeV} / \mathrm{MeV}$.

Fig. 5 The energy spectra of the electron from $\bar{B}^{0} \rightarrow D^{+} \pi^{0} e^{-} \bar{\nu}_{e}$ in the resonant region (solid line) and in the nonresonant region (broken lines). Shown are effects of two pion momentum cutoffs (100 MeV and $200 \mathrm{MeV}$ ) in the $D$-frame on the nonresonant contributions. The resonant contribution is not affected by these pion momentum cutoffs. The spectrum is in units of $\mathrm{MeV} / \mathrm{MeV}$.

Fig. 6 The energy spectra of the pion from $\bar{B}^{0} \rightarrow D^{+} \pi^{0} e^{-} \bar{\nu}_{e}$ in the resonant region (solid line) and in the nonresonant region (broken lines). Shown are effects of two pion momentum cutoffs (100 MeV and $200 \mathrm{MeV}$ ) in the $D$-frame on the nonresonant contributions. The resonant contribution is not affected by these pion momentum cutoffs. The spectrum is in units of $\mathrm{MeV} / \mathrm{MeV}$.

Fig. 7 The decay rates $\Gamma\left(\bar{B}^{0} \rightarrow D^{+} \pi^{0} e^{-} \bar{\nu}_{e}\right)$ (labeled as neutral pion) and $\Gamma\left(\bar{B}^{0} \rightarrow D^{0} \pi^{+} e^{-} \bar{\nu}_{e}\right)$ (labeled as charged pion) in the resonant region as a function of $1 / \Gamma_{D^{*}}$. The pion momentum cutoff of $100 \mathrm{MeV}$ or $200 \mathrm{MeV}$ in the $D$-frame has no effect on these rates.

Fig. 8 The decay rates $\Gamma\left(\bar{B}^{0} \rightarrow D^{+} \pi^{0} e^{-} \bar{\nu}_{e}\right)$ (labeled as neutral pion) and $\Gamma\left(\bar{B}^{0} \rightarrow D^{0} \pi^{+} e^{-} \bar{\nu}_{e}\right)$ (labeled as charged pion) in the nonresonant region as a function of $1 / \Gamma_{D^{*}}$. The pion momentum cutoff is $100 \mathrm{MeV}$ in the $D$-frame.

Fig. 9 The decay rates $\Gamma\left(B^{-} \rightarrow D^{0} \pi^{0} e^{-} \bar{\nu}_{e}\right)$ (labeled as neutral pion) in the resonant region as a function of $1 / \Gamma_{D^{*}}$. The other mode $B^{-} \rightarrow D^{+} \pi^{-} e^{-} \bar{\nu}_{e}$ is kinematically forbidden in the resonant region. The pion momentum cutoff of $100 \mathrm{MeV}$ or $200 \mathrm{MeV}$ in the $D$-frame has no effect on these rates. 
Fig. 10 The decay rates $\Gamma\left(B^{-} \rightarrow D^{0} \pi^{0} e^{-} \bar{\nu}_{e}\right)$ (labeled as neutral pion) and $\Gamma\left(B^{-} \rightarrow\right.$ $D^{+} \pi^{-} e^{-} \bar{\nu}_{e}$ ) (labeled as charged pion) in the nonresonant region as a function of $1 / \Gamma_{D^{*}}$. The pion momentum cutoff is $100 \mathrm{MeV}$ in the $D$-frame. See Appendix B for an explanation of the different behavior of the two decay rates.

Fig. 11 The energy spectra of the $D^{*}$ meson with different polarizations in the $B$-frame from $\bar{B}^{0} \rightarrow D^{*+} \pi^{0} e^{-} \bar{\nu}_{e}$ with a pion momentum cutoff of $100 \mathrm{MeV}$ in the $B$-frame.

Fig. 12 The energy spectra of the electron from $\bar{B}^{0} \rightarrow D^{*+} \pi^{0} e^{-} \bar{\nu}_{e}$ for different $D^{*}$ polarizations in the $B$-frame with a pion momentum cutoff of $100 \mathrm{MeV}$ in the $B$-frame.

Fig. 13 The energy spectra of the pion from $\bar{B}^{0} \rightarrow D^{*+} \pi^{0} e^{-} \bar{\nu}_{e}$ for different $D^{*}$ polarizations in the $B$-frame with a pion momentum cutoff of $100 \mathrm{MeV}$ in the $B$-frame.

Fig. 14 The energy spectra of the $D^{*}$ meson with different polarizations in the $B$-frame from $\bar{B}^{0} \rightarrow D^{*+} \pi^{0} e^{-} \bar{\nu}_{e}$ with a pion momentum cutoff of $100 \mathrm{MeV}$ in both the $B$ - and $D^{*}$-frames.

Fig. 15 The energy spectra of the electron from $\bar{B}^{0} \rightarrow D^{*+} \pi^{0} e^{-} \bar{\nu}_{e}$ for different $D^{*}$ polarizations in the $B$-frame with a pion momentum cutoff of $100 \mathrm{MeV}$ in both the $B$ - and $D^{*}$-frames.

Fig. 16 The energy spectra of the pion from $\bar{B}^{0} \rightarrow D^{*+} \pi^{0} e^{-} \bar{\nu}_{e}$ for different $D^{*}$ polarizations in the $B$-frame with a pion momentum cutoff of $100 \mathrm{MeV}$ in both the $B$ - and $D^{*}$-frames.

Fig. 17 The ratio $d \Gamma\left(\bar{B}^{0} \rightarrow D^{*+} \pi^{0} e^{-} \bar{\nu}\right) / d \Gamma\left(\bar{B}^{0} \rightarrow D^{+} \pi^{0} e^{-} \bar{\nu}\right)$ for different $D^{*}$ polarizations in the $B$-frame as a function of $v \cdot v^{\prime}$. The $D^{+} \pi^{0}$ system is in the resonant region. The pion momentum cutoff is $100 \mathrm{MeV}$ in both the $B$ - and $D^{*}(D)$-frame.

Fig. 18 The ratio $d \Gamma\left(\bar{B}^{0} \rightarrow D^{*+} \pi^{0} e^{-} \bar{\nu}\right) / d \Gamma\left(\bar{B}^{0} \rightarrow D^{+} \pi^{0} e^{-} \bar{\nu}\right)$ for different $D^{*}$ polarizations in the $B$-frame as a function of $v \cdot v^{\prime}$. The $D^{+} \pi^{0}$ system is in the nonresonant region. The pion momentum cutoff is $100 \mathrm{MeV}$ in both the $B$ - and $D^{*}(D)$-frame.

Fig. 19 The ratio $d \Gamma\left(\bar{B}^{0} \rightarrow D^{*+} \pi^{0} e^{-} \bar{\nu}\right) / d \Gamma\left(\bar{B}^{0} \rightarrow D^{+} \pi^{0} e^{-} \bar{\nu}\right)$ for different $D^{*}$ polarizations in the $B$-frame as a function of $v \cdot v^{\prime}$. The $D^{+} \pi^{0}$ system is in the resonant region. The pion momentum cutoff is $200 \mathrm{MeV}$ in both the $B$ - and $D^{*}(D)$-frame.

Fig. 20 The ratio $d \Gamma\left(\bar{B}^{0} \rightarrow D^{*+} \pi^{0} e^{-} \bar{\nu}\right) / d \Gamma\left(\bar{B}^{0} \rightarrow D^{+} \pi^{0} e^{-} \bar{\nu}\right)$ for different $D^{*}$ polarizations in the $B$-frame as a function of $v \cdot v^{\prime}$. The $D^{+} \pi^{0}$ system is in the nonresonant region. The pion momentum cutoff is $200 \mathrm{MeV}$ in both the $B$ - and $D^{*}(D)$-frame.

Fig. 21 The decay rates $\Gamma\left(\bar{B}^{0} \rightarrow D^{+} \pi^{0} e^{-} \bar{\nu}\right)$ for nonresonant $D^{+} \pi^{0}$ as a function of the pion momentum cutoff in the $D$-frame.

Fig. 22 The decay rates $\Gamma\left(\bar{B}^{0} \rightarrow D^{*+} \pi^{0} e^{-} \bar{\nu}\right)$ as a function of the pion momentum cutoff in the $B$-frame.

Fig. 23 The decay rates $\Gamma\left(\bar{B}^{0} \rightarrow D^{*+} \pi^{0} e^{-} \bar{\nu}\right)$ as a function of the pion momentum cutoff in both the $B$ - and $D^{*}$-frame. 